\title{
MAKING NONTRIVIALLY ASSOCIATED MODULAR CATEGORIES FROM FINITE GROUPS
}

\author{
M. M. AL-SHOMRANI and E. J. BEGGS
}

Received 15 August 2003

\begin{abstract}
We show that the double $\mathscr{D}$ of the nontrivially associated tensor category constructed from left coset representatives of a subgroup of a finite group $X$ is a modular category. Also we give a definition of the character of an object in this category as an element of a braided Hopf algebra in the category. This definition is shown to be adjoint invariant and multiplicative on tensor products. A detailed example is given. Finally, we show an equivalence of categories between the nontrivially associated double $\mathscr{D}$ and the trivially associated category of representations of the Drinfeld double of the group $D(X)$.
\end{abstract}

2000 Mathematics Subject Classification: 18D10, 16W30.

1. Introduction. This paper will make continual use of formulae and ideas from [2], and these definitions and formulae will not be repeated, as they would add very considerably to the length of the paper. The paper [2] is itself based on the papers $[3,4]$, but is mostly self-contained in terms of notation and definitions. The book [6] has been used as a standard reference for Hopf algebras, and [1, 8] as references for modular categories.

In [2], there is a construction of a nontrivially associated tensor category $\mathscr{C}$ from data which is a choice of left coset representatives $M$ for a subgroup $G$ of a finite group $X$. This introduces a binary operation "." and a $G$-valued "cocycle" $\tau$ on $M$. There is also a double construction where $X$ is viewed as a subgroup of a larger group. This gives rise to a braided category $\mathscr{D}$, which is the category of reps of an algebra $D$, which is itself in the category, and it is the category that we concentrate on in this paper.

It is our aim to show that the nontrivially associated algebra $D$ has reps which have characters in the same way that the reps of a finite group have characters, and also that the category of its representations has a modular structure in the same way that the category of reps of the double of a group has a modular structure.

We begin by describing the indecomposable objects in $\mathscr{C}$, in a manner similar to that used in [4]. A detailed example is given using the group $D_{6}$. Then we show how to find the dual objects in the category, and again illustrate this with an example.

Next, we show that the rigid braided category $\mathscr{D}$ is a ribbon category. The ribbon maps are calculated for the indecomposable objects in our example category.

In the next section, we explicitly evaluate in $\mathscr{D}$ the standard diagram for trace in a ribbon category [6]. Then we define the character of an object in $\mathscr{D}$ as an element of the dual of the braided Hopf algebra $D$. This element is shown to be right adjoint invariant. Also we show that the character is multiplicative for the tensor product of 
objects. A formula is found for the character in $\mathscr{D}$ in terms of characters of group representations.

The last ingredient needed for a modular category is the trace of the double braiding, and this is calculated in $\mathscr{D}$ in terms of group characters. Then the matrices $S, T$, and $C$, implementing the modular representation, are calculated explicitly in our example.

Finally, we show an equivalence of categories between the nontrivially associated double $\mathscr{D}$ and the category of representations of the Drinfeld double of the group $D(X)$.

Throughout the paper, we assume that all groups mentioned are finite, and that all vector spaces are finite-dimensional. We take the base field to be the complex numbers $\mathbb{C}$.

2. Indecomposable objects in $\mathscr{b}$. The objects of $\mathscr{C}$ are the right representations of the algebra $A$ described in [2]. We now look at the indecomposable objects in $\mathscr{b}$, or the irreducible representations of $A$, in a manner similar to that used in [4].

THEOREM 2.1. The indecomposable objects in $\mathscr{b}$ are of the form

$$
V=\bigoplus_{s \in \mathbb{O}} V_{s}
$$

where 0 is an orbit in $M$ under the $G$ action $\triangleleft$, and each $V_{s}$ is an irreducible right representation of the stabilizer of $s, \operatorname{stab}(s)$. Every object $T$ in $\mathscr{C}$ can be written as a direct sum of indecomposable objects in $\mathscr{b}$.

Proof. For an object $T$ in $\mathscr{C}$, we can use the $M$-grading to write

$$
T=\bigoplus_{s \in M} T_{s}
$$

but as $M$ is a disjoint union of orbits $O_{s}=\{s \triangleleft u: u \in G\}$ for $s \in M, T$ can be rewritten as a disjoint sum over orbits:

$$
T=\bigoplus_{0} T_{\odot}
$$

where

$$
T_{0}=\bigoplus_{s \in \mathbb{O}} T_{s}
$$

Now we will define the stabilizer of $s \in \mathbb{O}$, which is a subgroup of $G$, as

$$
\operatorname{stab}(s)=\{u \in G: s \triangleleft u=s\}
$$

As $\langle\eta \triangleleft u\rangle=\langle\eta\rangle \triangleleft u$ for all $\eta \in T, T_{s}$ is a representation of the group $\operatorname{stab}(s)$. Now fix a base point $t \in \mathcal{O}$. Because $\operatorname{stab}(t)$ is a finite group, $T_{t}$ is a direct sum of irreducible group representations $W_{i}$ for $i=1, \ldots, m$, that is,

$$
T_{t}=\bigoplus_{i=1}^{m} W_{i} .
$$


Suppose that $O=\left\{t_{1}, t_{2}, \ldots, t_{n}\right\}$, where $t_{1}=t$, and take $u_{i} \in G$ so that $t_{i}=t \triangleleft u_{i}$. Define

$$
U_{i}=\bigoplus_{j=1}^{n} W_{i} \bar{\triangleleft} u_{j} \subset \bigoplus_{s \in \mathbb{O}} T_{s} .
$$

We claim that each $U_{i}$ is an indecomposable object in $\mathscr{C}$. For any $v \in G$ and $\xi \bar{\triangleleft} u_{k} \in$ $W_{i} \triangleleft u_{k}$,

$$
\left(\xi \bar{\triangleleft} u_{k}\right) \bar{\triangleleft} v=\left(\xi \bar{\triangleleft}\left(u_{k} v u_{j}{ }^{-1}\right)\right) \bar{\triangleleft} u_{j},
$$

where $u_{k} v u_{j}^{-1} \in \operatorname{stab}(t)$ for some $u_{j} \in G$. This shows that $U_{i}$ is a representation of $G$. By the definition of $U_{i}$, any subrepresentation of $U_{i}$ which contains $W_{i}$ must be all of $U_{i}$. Thus $U_{i}$ is an indecomposable object in $\mathscr{C}$ and

$$
T_{\overparen{O}}=\bigoplus_{i=1}^{m} U_{i} .
$$

THEOREM 2.2 (Schur's lemma). Let $V$ and $W$ be two indecomposable objects in $b$ and let $\alpha: V \rightarrow W$ be a morphism. Then $\alpha$ is zero or a scalar multiple of the identity.

Proof. $V$ and $W$ are associated to orbits $\mathcal{O}$ and $\mathcal{O}^{\prime}$ so that $V=\bigoplus_{s \in \mathcal{O}} V_{s}$ and $W=$ $\bigoplus_{s \in \mathcal{O}^{\prime}} W_{s}$. As morphisms preserve grade, if $\alpha \neq 0$, then $\mathcal{O}=\mathcal{O}^{\prime}$. Now, if we take $s \in \mathbb{O}$, we will find that $\alpha: V_{s} \rightarrow W_{s}$ is a map of irreps of $\operatorname{stab}(s)$, so by Schur's lemma for groups, any nonzero map is a scalar multiple of the identity, and we have $V_{s}=W_{s}$ as representations of $\operatorname{stab}(s)$. Now we need to check that the multiple of the identity is the same for each $s \in \mathcal{O}$. Suppose that $\alpha$ is a multiplication by $\lambda$ on $V_{s}$. Given $t \in \mathcal{O}$, there is a $u \in G$ so that $t \triangleleft u=s$. Then, for $\eta \in V_{t}$,

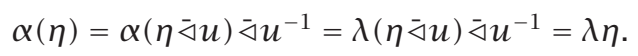

LEMMA 2.3. Let $V$ be an indecomposable object in $\mathscr{b}$ associated to the orbit $\mathbb{O}$. Choose $s, t \in \mathcal{O}$ and $u \in G$ so that $s \triangleleft u=t$. Then $V_{s}$ and $V_{t}$ are irreps of $\operatorname{stab}(s)$ and $\operatorname{stab}(t)$, respectively, and the group characters obey $\chi_{V_{t}}(v)=\chi_{V_{s}}\left(u v u^{-1}\right)$.

Proof. Note that $\bar{\triangleleft} u$ is an invertible map from $V_{s}$ to $V_{t}$. Then we have the commuting diagram

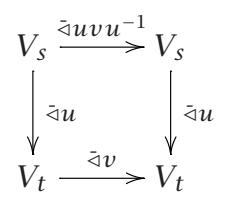

which implies that $\operatorname{trace}\left(\bar{\triangleleft} u v u^{-1}: V_{s} \rightarrow V_{s}\right)=\operatorname{trace}\left(\bar{\triangleleft} v: V_{t} \rightarrow V_{t}\right)$.

3. An example of indecomposable objects. We give an example of indecomposable objects in the categories discussed in the last section. As we will later want to have a 
TABLE 3.1

\begin{tabular}{rrrrrcrr}
\hline \multicolumn{2}{r}{ Irreps } & $\{e\}$ & $\left\{a^{3}\right\}$ & $\left\{b, b a^{2}, b a^{4}\right\}$ & $\left\{b a, b a^{3}, b a^{5}\right\}$ & $\left\{a^{2}, a^{4}\right\}$ & $\left\{a, a^{5}\right\}$ \\
\hline $1_{1}$ & 2 & 1 & 1 & 1 & 1 & 1 & 1 \\
$1_{2}$ & 22 & 1 & -1 & -1 & 1 & 1 & -1 \\
$1_{3}$ & 23 & 1 & -1 & 1 & -1 & 1 & -1 \\
$1_{4}$ & 2 & 1 & 1 & -1 & -1 & 1 & 1 \\
$1_{5}$ & 25 & 2 & -2 & 0 & 0 & -1 & 1 \\
$1_{6}$ & 26 & 2 & 2 & 0 & 0 & -1 & -1 \\
\hline
\end{tabular}

TABLE 3.2

\begin{tabular}{llllllll}
\hline \multicolumn{2}{c}{ Irreps } & $e$ & $a$ & $a^{2}$ & $a^{3}$ & $a^{4}$ & $a^{5}$ \\
\hline $3_{0}$ & $4_{0}$ & 1 & 1 & 1 & 1 & 1 & 1 \\
$3_{1}$ & $4_{1}$ & 1 & $\omega^{1}$ & $\omega^{2}$ & $\omega^{3}$ & $\omega^{4}$ & $\omega^{5}$ \\
$3_{2}$ & $4_{2}$ & 1 & $\omega^{2}$ & $\omega^{4}$ & 1 & $\omega^{2}$ & $\omega^{4}$ \\
$3_{3}$ & $4_{3}$ & 1 & $\omega^{3}$ & 1 & $\omega^{3}$ & 1 & $\omega^{3}$ \\
$3_{4}$ & $4_{4}$ & 1 & $\omega^{4}$ & $\omega^{2}$ & 1 & $\omega^{4}$ & $\omega^{2}$ \\
$3_{5}$ & $4_{5}$ & 1 & $\omega^{5}$ & $\omega^{4}$ & $\omega^{3}$ & $\omega^{2}$ & $\omega^{1}$ \\
\hline
\end{tabular}

category with braiding, we use the double construction in [2]. We also use Lemma 2.3 to list the group characters [5] for every point in the orbit in terms of the given base points.

Take $X$ to be the dihedral group $D_{6}=\left\langle a, b: a^{6}=b^{2}=e, a b=b a^{5}\right\rangle$, whose elements we list as $\left\{e, a, a^{2}, a^{3}, a^{4}, a^{5}, b, b a, b a^{2}, b a^{3}, b a^{4}, b a^{5}\right\}$, and $G$ to be the nonabelian normal subgroup of order 6 generated by $a^{2}$ and $b$, that is, $G=\left\{e, a^{2}, a^{4}, b, b a^{2}, b a^{4}\right\}$. We choose $M=\{e, a\}$. The center of $D_{6}$ is the subgroup $\left\{e, a^{3}\right\}$, and it has the following conjugacy classes: $\{e\},\left\{a^{3}\right\},\left\{a^{2}, a^{4}\right\},\left\{a, a^{5}\right\},\left\{b, b a^{2}, b a^{4}\right\}$, and $\left\{b a, b a^{3}, b a^{5}\right\}$.

The category $\mathscr{D}$ consists of right representations of the group $X=D_{6}$ which are graded by $Y=D_{6}$ (as a set), using the actions $\tilde{\triangleleft}: Y \times X \rightarrow Y$ and $\tilde{\triangleright}: Y \times X \rightarrow X$ which are defined as follows:

$$
y \tilde{\triangleleft} x=x^{-1} y x, \quad v t \tilde{\triangleright} x=v^{-1} x v^{\prime}=t x t^{\prime-1},
$$

for $x \in X, y \in Y, v, v^{\prime} \in G$, and $t, t^{\prime} \in M$, where $v t \tilde{\triangleleft} x=v^{\prime} t^{\prime}$.

Now let $V$ be an indecomposable object in $\mathscr{D}$. We get the following cases.

Case (1). Take the orbit $\{e\}$ with base point $e$, whose stabilizer is the whole of $D_{6}$. There are six possible irreducible group representations of the stabilizer, with their characters given by Table 3.1 [7].

Case (2). Take the orbit $\left\{a^{3}\right\}$ with base point $a^{3}$, whose stabilizer is the whole of $D_{6}$. There are six possible irreps $\left\{2_{1}, 2_{2}, 2_{3}, 2_{4}, 2_{5}, 2_{6}\right\}$, with characters given by Table 3.1 .

Case (3). Take the orbit $\left\{a^{2}, a^{4}\right\}$ with base point $a^{2}$, whose stabilizer is $\left\{e, a, a^{2}, a^{3}\right.$, $\left.a^{4}, a^{5}\right\}$. There are six irreps $\left\{3_{0}, 3_{1}, 3_{2}, 3_{3}, 3_{4}, 3_{5}\right\}$, with characters given by Table 3.2 , where $\omega=e^{i \pi / 3}$. Applying Lemma 2.3 gives $\chi_{V_{a^{4}}}(v)=\chi_{V_{a^{2}}}(b v b)$. 
TABLE 3.3

\begin{tabular}{ccrrr}
\hline Irreps & $e$ & $a^{3}$ & \multicolumn{1}{c}{$b$} & $b a^{3}$ \\
\hline $5_{++}$ & 1 & 1 & 1 & 1 \\
$5_{+-}$ & 1 & 1 & -1 & -1 \\
$5_{-+}$ & 1 & -1 & 1 & -1 \\
$5_{--}$ & 1 & -1 & -1 & 1 \\
\hline
\end{tabular}

TABLE 3.4

\begin{tabular}{ccrrr}
\hline Irreps & $e$ & $a^{3}$ & $b a$ & $b a^{4}$ \\
\hline $6_{++}$ & 1 & 1 & 1 & 1 \\
$6_{-+}$ & 1 & -1 & 1 & -1 \\
$6_{+-}$ & 1 & 1 & -1 & -1 \\
$6_{--}$ & 1 & -1 & -1 & 1 \\
\hline
\end{tabular}

Case (4). Take the orbit $\left\{a, a^{5}\right\}$ with base point $a$, whose stabilizer is $\left\{e, a, a^{2}, a^{3}\right.$, $\left.a^{4}, a^{5}\right\}$. There are six irreps $\left\{4_{0}, 4_{1}, 4_{2}, 4_{3}, 4_{4}, 4_{5}\right\}$ with characters given in Table 3.2. Applying Lemma 2.3 gives $\chi_{V_{a^{5}}}(v)=\chi_{V a}\left(b a^{2} v b a^{2}\right)$.

Case (5). Take the orbit $\left\{b, b a^{2}, b a^{4}\right\}$ with base point $b$, whose stabilizer is $\left\{e, a^{3}\right.$, $\left.b, b a^{3}\right\}$. There are four irreps with characters given by Table 3.3. Applying Lemma 2.3 gives $\chi_{V_{b a^{2}}}(v)=\chi_{V_{b}}\left(a^{4} v a^{2}\right)$ and $\chi_{v_{b a^{4}}}(v)=\chi_{V_{b}}\left(a^{2} v a^{4}\right)$.

Case (6). Take the orbit $\left\{b a, b a^{3}, b a^{5}\right\}$ with base point $b a$, whose stabilizer is $\left\{e, a^{3}\right.$, $\left.b a, b a^{4}\right\}$. There are four irreps with characters given by Table 3.4. Applying Lemma 2.3 gives $\chi_{V_{b a^{3}}}(v)=\chi_{V_{b a}}\left(a^{4} v a^{2}\right)$ and $\chi_{V_{b a^{5}}}(v)=\chi_{V_{b a}}\left(a^{2} v a^{4}\right)$.

4. Duals of indecomposable objects in $\mathscr{C}$. Given an irreducible object $V$ with associated orbit $\mathbb{O}$ in $\mathscr{C}$, how do we find its dual $V^{*}$ ? The dual would be described, as in Section 2, by an orbit, a base point in the orbit, and a right group representation of the stabilizer of the base point. Using the formula $\left(s^{L} \cdot s\right) \triangleleft u=\left(s^{L} \triangleleft(s \triangleright u)\right) \cdot(s \triangleleft u)=e$, we see that the left inverse of a point in the orbit containing $s$ is in the orbit containing $s^{L}$. By using the evaluation map from $V^{*} \otimes V$ to the field, we can take $\left(V^{*}\right)_{s^{L}}=\left(V_{s}\right)^{*}$ as vec-

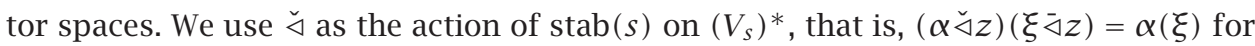

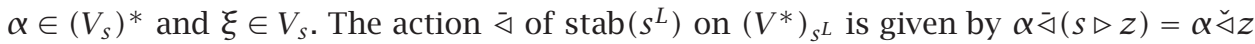
for $z \in \operatorname{stab}(s)$. In terms of group characters, this gives

$$
\chi_{\left(V^{*}\right)_{s} L}(s \triangleright z)=\chi_{\left(V_{s}\right)^{*}}(z), \quad z \in \operatorname{stab}(s) .
$$

If we take $\mathcal{O}^{L}=\left\{s^{L}: s \in \mathcal{O}\right\}$ to have base point $p$, and choose $u \in G$ so that $p \triangleleft u=s^{L}$, then using Lemma 2.3 gives

$$
\chi_{\left(V^{*}\right)_{s} L}(s \triangleright z)=\chi_{\left(V_{s}\right)^{*}}(z)=\chi_{\left(V^{*}\right) p}\left(u(s \triangleright z) u^{-1}\right), \quad z \in \operatorname{stab}(s) .
$$

This formula allows us to find the character of $V^{*}$ at its base point $p$ as a representation of $\operatorname{stab}(p)$ in terms of the character of the dual of $V_{s}$ as a representation of $\operatorname{stab}(s)$. 
LEMMA 4.1. In $\mathscr{b},(V \otimes W)^{*}$ can be regarded as $W^{*} \otimes V^{*}$ with the evaluation

$$
(\alpha \otimes \beta)(\xi \otimes \eta)=(\alpha \bar{\triangleleft} \tau(\langle\beta\rangle,\langle\xi\rangle \cdot\langle\eta\rangle))(\eta)\left(\beta \bar{\triangleleft} \tau(\langle\xi\rangle,\langle\eta\rangle)^{-1}\right)(\xi) .
$$

Given a basis $\{\xi\}$ of $V$ and a basis $\{\eta\}$ of $W$, the dual basis $\{\widehat{\xi \otimes \eta}\}$ of $W^{*} \otimes V^{*}$ can be written in terms of the dual basis of $V^{*}$ and $W^{*}$ as

$$
\widehat{\xi \otimes \eta}=\hat{\eta} \triangleleft \tau\left(\langle\xi\rangle^{L} \triangleleft \tau(\langle\xi\rangle,\langle\eta\rangle),\langle\xi\rangle \cdot\langle\eta\rangle\right)^{-1} \otimes \hat{\xi} \triangleleft \tau(\langle\xi\rangle,\langle\eta\rangle) .
$$

Proof. Applying the associator to $(\alpha \otimes \beta) \otimes(\xi \otimes \eta)$ gives

$$
\alpha \bar{\triangleleft} \tau(\langle\beta\rangle,\langle\xi\rangle \cdot\langle\eta\rangle) \otimes(\beta \otimes(\xi \otimes \eta)),
$$

and then applying the inverse associator gives

$$
\alpha \bar{\triangleleft}(\langle\beta\rangle,\langle\xi\rangle \cdot\langle\eta\rangle) \otimes\left(\left(\beta \bar{\triangleleft} \tau(\langle\xi\rangle,\langle\eta\rangle)^{-1} \otimes \xi\right) \otimes \eta\right) .
$$

Applying the evaluation map first to $\beta \bar{\triangleleft} \tau(\langle\xi\rangle,\langle\eta\rangle)^{-1} \otimes \xi$ then to $\alpha \bar{\triangleleft} \tau(\langle\beta\rangle,\langle\xi\rangle \cdot\langle\eta\rangle) \otimes \eta$ gives the first equation. For the evaluation to be nonzero, we need $\left(\langle\beta\rangle \triangleleft \tau(\langle\xi\rangle,\langle\eta\rangle)^{-1}\right)$. $\langle\xi\rangle=e$ which implies $\langle\beta\rangle \triangleleft \tau(\langle\xi\rangle,\langle\eta\rangle)^{-1}=\langle\xi\rangle^{L}$ or, equivalently, $\langle\beta\rangle=\langle\xi\rangle^{L} \triangleleft \tau(\langle\xi\rangle,\langle\eta\rangle)$. This gives the second equation.

EXAMPLE 4.2. Using (4.2), we calculate the duals of the objects given in the last section.

Case (1). The orbit $\{e\}$ has left inverse $\{e\}$, so $\chi_{\left(V^{*}\right)_{e}}=\chi_{\left(V_{e}\right)^{*}}$. By a calculation with group characters, all the listed irreps of $\operatorname{stab}(e)$ are self-dual, so $1_{r}^{*}=1_{r}$ for $r \in$ $\{1, \ldots, 6\}$.

Case (2). The orbit $\left\{a^{3}\right\}$ has left inverse $\left\{a^{3}\right\}$, so $\chi_{\left(V^{*}\right)} a^{3}=\chi_{\left(V_{a^{3}}\right)}$. As in the last case, the group representations are self-dual, so $2_{r}^{*}=2_{r}$ for $r \in\{1, \ldots, 6\}$.

Case (3). The left inverse of the base point $a^{2}$ is $a^{4}$, which is still in the orbit. As group representations, the dual of $3_{r}$ is $3_{6-r}(\bmod 6)$. Applying Lemma 2.3 to move the base point, we see that the dual of $3_{r}$ in the category is $3_{r}$.

Case (4). The left inverse of the base point $a$ is $a^{5}$, which is still in the orbit. As in the last case, the dual of $4_{r}$ in the category is $4_{r}$.

Case (5). The left inverse of the base point is itself, and as group representations, all Case (5) irreps are self-dual. We deduce that in the category the objects are self-dual.

Case (6). Self-dual as in Case (5).

\section{The ribbon map on the category $\mathscr{D}$}

THEOREM 5.1. The ribbon transformation $\theta_{V}: V \rightarrow V$ for any object $V$ in $\mathscr{D}$ can be defined by $\theta_{V}(\xi)=\xi \hat{\triangleleft}\|\xi\|$.

Proof. In the following lemmas, we show that the required properties hold.

LEMMA 5.2. $\theta_{V}$ is a morphism in the category. 
Proof. Begin by checking the $X$-grade: for $\xi \in V$,

$$
\left\|\theta_{V}(\xi)\right\|=\|\xi \hat{\triangleleft}\| \xi\|\|=\|\xi\| \tilde{\triangleleft}\|\xi\|=\|\xi\| .
$$

Now we check the $X$-action, that is, that $\theta_{V}(\xi \hat{\triangleleft} x)=\theta_{V}(\xi) \hat{\triangleleft} x$ :

$$
\begin{aligned}
\theta_{V}(\xi \hat{\triangleleft} x) & =(\xi \hat{\triangleleft} x) \hat{\triangleleft}\|\xi \hat{\triangleleft} x\|=(\xi \hat{\triangleleft} x) \hat{\triangleleft}(\|\xi\| \tilde{\triangleleft} x) \\
& =\xi \hat{\triangleleft} x x^{-1}\|\xi\| x=(\xi \hat{\triangleleft}\|\xi\|) \hat{\triangleleft} x=\theta_{V}(\xi) \hat{\triangleleft} x .
\end{aligned}
$$

LEMMA 5.3. For any two objects $V$ and $W$ in $\mathscr{D}$,

$$
\theta_{V \otimes W}=\Psi_{V \otimes W}^{-1} \circ \Psi_{W \otimes V}^{-1} \circ\left(\theta_{V} \otimes \theta_{W}\right)=\left(\theta_{V} \otimes \theta_{W}\right) \circ \Psi_{V \otimes W}^{-1} \circ \Psi_{W \otimes V}^{-1} .
$$

This can also be described by the following:
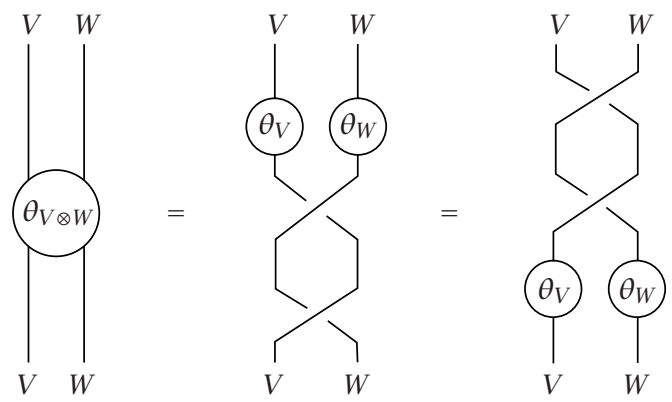

Proof. First calculate $\Psi(\Psi(\xi \otimes \eta))$ for $\xi \in V$ and $\eta \in W$, beginning with

$$
\Psi(\Psi(\xi \otimes \eta))=\Psi\left(\eta \hat{\triangleleft}(\langle\xi\rangle \triangleleft|\eta|)^{-1} \otimes \xi \hat{\triangleleft}|\eta|\right) .
$$

To simplify what follows, we will use the substitutions

$$
\eta^{\prime}=\xi \hat{\triangleleft}|\eta|, \quad \xi^{\prime}=\eta \hat{\triangleleft}(\langle\xi\rangle \triangleleft|\eta|)^{-1},
$$

so (5.5) can be rewritten as

$$
\Psi(\Psi(\xi \otimes \eta))=\Psi\left(\xi^{\prime} \otimes \eta^{\prime}\right)=\eta^{\prime} \triangleleft\left(\left\langle\xi^{\prime}\right\rangle \triangleleft\left|\eta^{\prime}\right|\right)^{-1} \otimes \xi^{\prime} \triangleleft\left|\eta^{\prime}\right| .
$$

As $\eta^{\prime}=\xi \triangleleft|\eta|=\xi \triangleleft|\eta|$, then $\left|\eta^{\prime}\right|=|\xi \triangleleft| \eta||=(\langle\xi\rangle \triangleright|\eta|)^{-1}|\xi||\eta|$, so

$$
\begin{aligned}
\xi^{\prime} \hat{\triangleleft}\left|\eta^{\prime}\right| & =\eta \hat{\triangleleft}(\langle\xi\rangle \triangleleft|\eta|)^{-1}(\langle\xi\rangle \triangleright|\eta|)^{-1}|\xi||\eta| \\
& =\eta \hat{\triangleleft}((\langle\xi\rangle \triangleright|\eta|)(\langle\xi\rangle \triangleleft|\eta|))^{-1}|\xi||\eta| \\
& =\eta \hat{\triangleleft}|\eta|^{-1}\langle\xi\rangle^{-1}|\xi||\eta| .
\end{aligned}
$$

Hence, if we put $y=\|\xi \otimes \eta\|=\|\xi\| \circ\|\eta\|=|\eta|^{-1}|\xi|^{-1}\langle\xi\rangle\langle\eta\rangle$,

$$
\Psi(\Psi(\xi \otimes \eta)) \hat{\triangleleft}\|\xi \otimes \eta\|=\xi \hat{\triangleleft}|\eta|\left(\left\langle\xi^{\prime}\right\rangle \triangleleft\left|\eta^{\prime}\right|\right)^{-1}(p \tilde{\triangleright}\|\xi \otimes \eta\|) \otimes \eta \hat{\triangleleft}|\eta|^{-1}\langle\eta\rangle,
$$


where, using (5.8),

$$
\begin{gathered}
p=\left\|\xi^{\prime} \hat{\triangleleft}\left|\eta^{\prime}\right|\right\|=\left|\xi^{\prime} \triangleleft\right| \eta^{\prime}||^{-1}\left\langle\xi^{\prime} \triangleleft\left|\eta^{\prime}\right|\right\rangle=\|\eta\| \tilde{\triangleleft}\|\eta\| y^{-1}=\|\eta\| \tilde{\triangleleft} y^{-1}, \\
p \tilde{\triangleright}\|\xi \otimes \eta\|=\left(\|\eta\| \tilde{\triangleleft} y^{-1}\right) \tilde{\triangleright} y=\left(\|\eta\| \tilde{\triangleright} y^{-1}\right)^{-1} .
\end{gathered}
$$

As $\left\|\xi^{\prime} \triangleleft\left|\eta^{\prime}\right|\right\|=v^{\prime} t^{\prime}=\|\eta\| \tilde{\triangleleft} y^{-1}$, by unique factorization, $t^{\prime}=\left\langle\xi^{\prime}\right\rangle \triangleleft\left|\eta^{\prime}\right|$. Then $\|\eta\| \tilde{\triangleright} y^{-1}$ $=\langle\eta\rangle y^{-1} t^{\prime-1}$, which implies that

$$
|\eta|\left(\left\langle\xi^{\prime}\right\rangle \triangleleft\left|\eta^{\prime}\right|\right)^{-1}\left(\|\eta\| \tilde{\triangleright} y^{-1}\right)^{-1}=|\eta| t^{\prime-1} t^{\prime} y\langle\eta\rangle^{-1}=\|\xi\| .
$$

Substituting this into (5.9) gives

$$
\Psi(\Psi(\xi \otimes \eta)) \hat{\triangleleft}\|\xi \otimes \eta\|=\xi \hat{\triangleleft}\|\xi\| \otimes \eta \hat{\triangleleft}\|\eta\| .
$$

LEMMA 5.4. For the unit object $\underline{\mathbf{1}}=\mathbb{C}$ in $\mathscr{D}, \theta_{1}$ is the identity.

Proof. For any object $V$ in $\mathscr{D}, \theta_{V}: V \rightarrow V$ is defined by

$$
\theta_{V}(\xi)=\xi \hat{\triangleleft}\|\xi\| \quad \text { for } \xi \in V \text {. }
$$

If we choose $V=\underline{\mathbf{1}}=\mathbb{C}$, then $\theta_{\underline{1}}(\xi)=\xi \hat{\triangleleft} e=\xi$ as $\|\xi\|=e$.

LEMMA 5.5. For any object $V$ in $\mathscr{D},\left(\theta_{V}\right)^{*}=\theta_{V^{*}}$ :

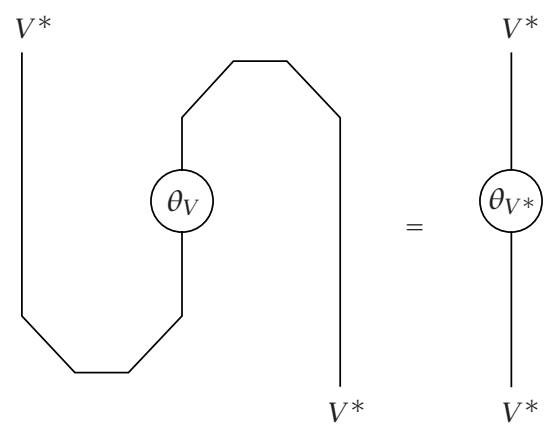

Proof. Begin with

$$
\begin{aligned}
\operatorname{coev}_{V}(1) & =\sum_{\xi \in \text { basis of } V} \xi \hat{\triangleleft} \tilde{\tau}\left(\|\xi\|^{L},\|\xi\|\right)^{-1} \otimes \hat{\xi} \\
& =\sum_{\xi \in \text { basis of } V} \xi \hat{\triangleleft} \tau\left(\langle\xi\rangle^{L},\langle\xi\rangle\right)^{-1} \otimes \hat{\xi} .
\end{aligned}
$$

For $\alpha \in V^{*}$, we follow (5.14) and calculate

$$
\left(\theta_{V}\right)^{*}(\alpha)=\left(\operatorname{eval}_{V} \otimes \mathrm{id}\right) \sum_{\xi \in \text { basis of } V} \Phi^{-1}\left(\alpha \otimes\left(\theta_{V}\left(\xi \hat{\triangleleft} \tau\left(\langle\xi\rangle^{L},\langle\xi\rangle\right)^{-1}\right) \otimes \hat{\xi}\right)\right) .
$$


Now, as $\tau\left(\langle\xi\rangle^{L},\langle\xi\rangle\right)=\langle\xi\rangle^{L}\langle\xi\rangle$,

$$
\begin{aligned}
\left\|\xi \hat{\triangleleft} \tau\left(\langle\xi\rangle^{L},\langle\xi\rangle\right)^{-1}\right\| & =\|\xi\| \tilde{\triangleleft}\left(\langle\xi\rangle^{L}\langle\xi\rangle\right)^{-1} \\
& =\langle\xi\rangle^{L}\langle\xi\rangle|\xi|^{-1}\langle\xi\rangle\langle\xi\rangle^{-1}\langle\xi\rangle^{L-1} \\
& =\langle\xi\rangle^{L}\langle\xi\rangle|\xi|^{-1}\langle\xi\rangle^{L-1}, \\
\theta_{V}\left(\xi \hat{\triangleleft} \tau\left(\langle\xi\rangle^{L},\langle\xi\rangle\right)^{-1}\right) & =\left(\xi \hat{\triangleleft} \tau\left(\langle\xi\rangle^{L},\langle\xi\rangle\right)^{-1}\right) \hat{\triangleleft}\left\|\xi \hat{\triangleleft} \tilde{\tau}\left(\|\xi\|^{L},\|\xi\|\right)^{-1}\right\| \\
& =\xi \hat{\triangleleft}\langle\xi\rangle^{-1}\langle\xi\rangle^{L-1}\langle\xi\rangle^{L}\langle\xi\rangle|\xi|^{-1}\langle\xi\rangle^{L-1} \\
& =\xi \hat{\triangleleft}|\xi|^{-1}\langle\xi\rangle^{L-1} .
\end{aligned}
$$

The next step is to find

$$
\begin{aligned}
& \Phi^{-1}\left(\alpha \otimes\left(\left(\xi \hat{\triangleleft}|\xi|^{-1}\langle\xi\rangle^{L-1}\right) \otimes \hat{\xi}\right)\right) \\
& \quad=\left(\alpha \hat{\triangleleft} \tilde{\tau}\left(|| \xi \hat{\triangleleft}|\xi|^{-1}\langle\xi\rangle^{L-1}\|,\| \hat{\xi} \|\right)^{-1} \otimes\left(\xi \hat{\triangleleft}|\xi|^{-1}\langle\xi\rangle^{L-1}\right)\right) \otimes \hat{\xi} .
\end{aligned}
$$

As

$$
\begin{aligned}
\left\|\xi \hat{\triangleleft}|\xi|^{-1}\langle\xi\rangle^{L-1}\right\| \\
\quad=\|\xi\| \tilde{\triangleleft}|\xi|^{-1}\langle\xi\rangle^{L-1} \\
=\langle\xi\rangle^{L}|\xi||\xi|^{-1}\langle\xi\rangle|\xi|^{-1}\langle\xi\rangle^{L-1} \\
\quad=\tau\left(\langle\xi\rangle^{L},\langle\xi\rangle\right)|\xi|^{-1}\langle\xi\rangle^{L-1} \\
=\tau\left(\langle\xi\rangle^{L},\langle\xi\rangle\right)|\xi|^{-1}\langle\xi\rangle \tau\left(\langle\xi\rangle^{L},\langle\xi\rangle\right)^{-1} \\
=\tau\left(\langle\xi\rangle^{L},\langle\xi\rangle\right)|\xi|^{-1}\left(\langle\xi\rangle \triangleright \tau\left(\langle\xi\rangle^{L},\langle\xi\rangle\right)^{-1}\right)\left(\langle\xi\rangle \triangleleft \tau\left(\langle\xi\rangle^{L},\langle\xi\rangle\right)^{-1}\right),
\end{aligned}
$$

then, as $\|\hat{\xi}\|=\|\xi\|^{L}=|\xi| \tau\left(\langle\xi\rangle^{L},\langle\xi\rangle\right)^{-1}\langle\xi\rangle^{L}$,

$$
\begin{aligned}
\Phi^{-1} & \left(\alpha \otimes\left(\left(\xi \hat{\triangleleft}|\xi|^{-1}\langle\xi\rangle^{L-1}\right) \otimes \hat{\xi}\right)\right) \\
& =\left(\alpha \hat{\triangleleft} \tau\left(\langle\xi\rangle \triangleleft \tau\left(\langle\xi\rangle^{L},\langle\xi\rangle\right)^{-1},\langle\xi\rangle^{L}\right)^{-1} \otimes\left(\xi \hat{\triangleleft}|\xi|^{-1}\langle\xi\rangle^{L-1}\right)\right) \otimes \hat{\xi} .
\end{aligned}
$$

Put $v=\tau\left(\langle\xi\rangle^{L},\langle\xi\rangle\right)^{-1}=\langle\xi\rangle^{-1}\langle\xi\rangle^{L-1}$ and $w=\tau\left(\langle\xi\rangle \triangleleft v,\langle\xi\rangle^{L}\right)^{-1}=\left((\langle\xi\rangle \triangleleft v)\langle\xi\rangle^{L}\right)^{-1}$; then substituting in (5.16) gives

$$
\left(\theta_{V}\right)^{*}(\alpha)=\left(\mathrm{eval}_{V} \otimes \mathrm{id}\right) \sum_{\xi \in \text { basis of } V}\left((\alpha \hat{\triangleleft} w) \otimes\left(\xi \hat{\triangleleft}|\xi|^{-1}\langle\xi\rangle^{L-1}\right)\right) \otimes \hat{\xi} .
$$

For a given term in the sum to be nonzero, we require that

$$
\|\alpha\|=\|\hat{\xi}\|=\|\xi\|^{L}=|\xi|\langle\xi\rangle^{-1},
$$

and we proceed under this assumption. Now calculate

$$
\operatorname{eval}_{V}\left((\alpha \hat{\triangleleft} w) \otimes\left(\xi \hat{\triangleleft}|\xi|^{-1}\langle\xi\rangle^{L-1}\right)\right)=(\beta \hat{\triangleleft}(\|\xi\| \tilde{\triangleright} p))(\xi \tilde{\triangleleft} p)=\beta(\xi),
$$


where $p=|\xi|^{-1}\langle\xi\rangle^{L-1}$ and $\beta=\alpha \hat{\triangleleft} w(\|\xi\| \tilde{\triangleright} p)^{-1}$. Next, we want to find $\|\xi\| \tilde{\triangleright} p$. To do this, we first find

$$
\begin{aligned}
\|\xi\| \tilde{\triangleleft} p & =\langle\xi\rangle^{L}|\xi||\xi|^{-1}\langle\xi\rangle|\xi|^{-1}\langle\xi\rangle^{L-1} \\
& =v^{-1}|\xi|^{-1}\langle\xi\rangle v=v^{-1}|\xi|^{-1}(\langle\xi\rangle \triangleright v)(\langle\xi\rangle \triangleleft v),
\end{aligned}
$$

and hence

$$
\begin{aligned}
\|\xi\| \tilde{\triangleright} p & =\langle\xi\rangle p(\langle\xi\rangle \triangleleft v)^{-1} \\
& =\langle\xi\rangle|\xi|^{-1}\langle\xi\rangle v(\langle\xi\rangle \triangleleft v)^{-1} \\
& =\langle\xi\rangle|\xi|^{-1}(\langle\xi\rangle \triangleright v) .
\end{aligned}
$$

Thus

$$
\begin{aligned}
\beta & =\alpha \hat{\triangleleft} w(\langle\xi\rangle \triangleright v)^{-1}|\xi|\langle\xi\rangle^{-1} \\
& =\alpha \hat{\triangleleft}\langle\xi\rangle^{L-1}(\langle\xi\rangle \triangleleft v)^{-1}(\langle\xi\rangle \triangleright v)^{-1}|\xi|\langle\xi\rangle^{-1} \\
& =\alpha \hat{\triangleleft}\langle\xi\rangle v(\langle\xi\rangle v)^{-1}|\xi|\langle\xi\rangle^{-1}=\alpha \hat{\triangleleft}|\xi|\langle\xi\rangle^{-1}
\end{aligned}
$$

Now, substituting these last equations in (5.21) gives

$$
\left(\theta_{V}\right)^{*}(\alpha)=\sum_{\xi \in \text { basis of } V,|\xi|\langle\xi\rangle^{-1}=\|\alpha\|}(\alpha \hat{\triangleleft}\|\alpha\|)(\xi) \cdot \hat{\xi} \text {. }
$$

Take a basis $\xi_{1}, \xi_{2}, \ldots, \xi_{n}$ with $(\alpha \hat{\triangleleft}\|\alpha\|)\left(\xi_{i}\right)$ being 1 if $i=1$, and 0 otherwise. Then

$$
\left(\theta_{V}\right)^{*}(\alpha)=\hat{\xi}_{1}+0=\alpha \hat{\triangleleft}\|\alpha\|=\theta_{V^{*}}(\alpha),
$$

where $\hat{\xi}_{1}, \hat{\xi}_{2}, \ldots, \hat{\xi}_{n}$ is the dual basis of $V^{*}$ defined by $\hat{\xi}_{i}\left(\xi_{j}\right)=\delta_{i, j}$.

EXAMPLE 5.6. We return to the example of Section 3. First, we calculate the value of the ribbon map on the indecomposable objects. For an irreducible representation $V$, we have $\theta_{V}: V \rightarrow V$ defined by $\theta_{V}(\xi)=\xi \hat{\triangleleft}\|\xi\|$ for $\xi \in V$. At the base point $s \in \mathcal{O}$, we have $\theta_{V}(\xi)=\xi \bar{\triangleleft} s$ for $\xi \in V$ and $\theta: V_{s} \rightarrow V_{s}$ is a multiple $\Theta_{V}$, say, of the identity or, more $\operatorname{explicitly,~} \operatorname{trace}\left(\theta: V_{s} \rightarrow V_{s}\right)=\Theta_{V} \operatorname{dim}_{\mathbb{C}}\left(V_{S}\right)$, that is,

$$
\Theta_{V}=\frac{\text { group character }(s)}{\operatorname{dim}_{\mathbb{C}}\left(V_{s}\right)}
$$

And then, for the different cases we will get Table 5.1. 
TABLE 5.1

\begin{tabular}{cccc}
\hline Irreps & $\Theta_{V}$ & Irreps & $\Theta_{V}$ \\
\hline $1_{1}$ & 1 & $3_{4}$ & $\omega^{2}$ \\
$1_{2}$ & 1 & $3_{5}$ & $\omega^{4}$ \\
$1_{3}$ & 1 & $4_{0}$ & 1 \\
$1_{4}$ & 1 & $4_{1}$ & $\omega^{1}$ \\
$1_{5}$ & 1 & $4_{2}$ & $\omega^{2}$ \\
$1_{6}$ & 1 & 43 & -1 \\
$2_{1}$ & 1 & $4_{4}$ & $\omega^{4}$ \\
$2_{2}$ & -1 & 45 & $\omega^{5}$ \\
$2_{3}$ & -1 & $5_{++}$ & 1 \\
$2_{4}$ & 1 & $5_{+-}$ & -1 \\
$2_{5}$ & -1 & $5_{-+}$ & 1 \\
$2_{6}$ & 1 & $5_{--}$ & -1 \\
$3_{0}$ & 1 & $6_{++}$ & 1 \\
$3_{1}$ & $\omega^{2}$ & $6_{-+}$ & 1 \\
$3_{2}$ & $\omega^{4}$ & $6_{+-}$ & -1 \\
$3_{3}$ & 1 & $6_{--}$ & -1 \\
\hline
\end{tabular}

\section{Traces in the category $\mathscr{D}$}

DEFINITION 6.1 [8]. The trace of a morphism $T: V \rightarrow V$ for any object $V$ in $\mathscr{D}$ is defined by the following diagram:

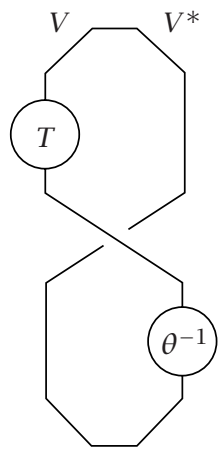

THEOREM 6.2. If the diagram of Definition 6.1 is evaluated in $\mathscr{D}$, the following is found:

$$
\operatorname{trace}(T)=\sum_{\xi \in \text { basis of } V} \hat{\xi}(T(\xi))
$$

Proof. Begin with

$$
\begin{aligned}
\operatorname{coev}_{V}(1) & =\sum_{\xi \in \text { basis of } V} \xi \hat{\triangleleft} \tilde{\tau}\left(\|\xi\|^{L},\|\xi\|^{-1} \otimes \hat{\xi}\right. \\
& =\sum_{\xi \in \text { basis of } V} \xi \hat{\triangleleft} \tau\left(\langle\xi\rangle^{L},\langle\xi\rangle\right)^{-1} \otimes \hat{\xi},
\end{aligned}
$$


and applying $T \otimes$ id to this gives

$$
\sum_{\xi \in \text { basis of } V} T\left(\xi \hat{\triangleleft} \tau\left(\langle\xi\rangle^{L},\langle\xi\rangle\right)^{-1}\right) \otimes \hat{\xi}=\sum_{\xi \in \text { basis of } V} T(\xi) \hat{\triangleleft} \tau\left(\langle\xi\rangle^{L},\langle\xi\rangle\right)^{-1} \otimes \hat{\xi} .
$$

Next, apply the braiding map to the last equation to get

$$
\sum_{\xi \in \text { basis of } V} \Psi\left(T(\xi) \hat{\triangleleft} \boldsymbol{T}\left(\langle\xi\rangle^{L},\langle\xi\rangle\right)^{-1} \otimes \hat{\xi}\right)=\sum_{\xi \in \text { basis of } V} \hat{\xi} \hat{\triangleleft}\left(\left\langle\xi^{\prime}\right\rangle \triangleleft|\hat{\xi}|\right)^{-1} \otimes \xi^{\prime} \hat{\triangleleft}|\hat{\xi}|,
$$

where $\xi^{\prime}=T(\xi) \hat{\triangleleft} \tau\left(\langle\xi\rangle^{L},\langle\xi\rangle\right)^{-1}$, so

$$
\begin{aligned}
\left\langle\xi^{\prime}\right\rangle & =\left\langle T(\xi) \triangleleft \tau\left(\langle\xi\rangle^{L},\langle\xi\rangle\right)^{-1}\right\rangle=\left\langle T(\xi) \triangleleft \tau\left(\langle\xi\rangle^{L},\langle\xi\rangle\right)^{-1}\right\rangle \\
& =\langle T(\xi)\rangle \triangleleft \tau\left(\langle\xi\rangle^{L},\langle\xi\rangle\right)^{-1}=\langle\xi\rangle \triangleleft \tau\left(\langle\xi\rangle^{L},\langle\xi\rangle\right)^{-1} .
\end{aligned}
$$

To calculate $|\hat{\xi}|$, we start with

$$
\|\hat{\xi}\|=\|\xi\|^{L}=\left(|\xi|^{-1}\langle\xi\rangle\right)^{L}=|\xi| \tau\left(\langle\xi\rangle^{L},\langle\xi\rangle\right)^{-1}\langle\xi\rangle^{L},
$$

which implies that $|\hat{\xi}|=\tau\left(\langle\xi\rangle^{L},\langle\xi\rangle\right)|\xi|^{-1}$. Then

$$
\begin{aligned}
& \hat{\xi} \triangleleft\left(\left\langle\xi^{\prime}\right\rangle \triangleleft|\hat{\xi}|\right)^{-1}=\hat{\xi} \triangleleft\left(\langle\xi\rangle \triangleleft \tau\left(\langle\xi\rangle^{L},\langle\xi\rangle\right)^{-1} \tau\left(\langle\xi\rangle^{L},\langle\xi\rangle\right)|\xi|^{-1}\right)^{-1} \\
&=\hat{\xi} \triangleleft\left(\langle\xi\rangle \triangleleft|\xi|^{-1}\right)^{-1}, \\
& \xi^{\prime} \triangleleft|\hat{\xi}|=\left(T(\xi) \hat{\triangleleft} \tau\left(\langle\xi\rangle^{L},\langle\xi\rangle\right)^{-1}\right) \hat{\triangleleft}\left(\tau\left(\langle\xi\rangle^{L},\langle\xi\rangle\right)|\xi|^{-1}\right)=T(\xi) \hat{\triangleleft}|\xi|^{-1},
\end{aligned}
$$

which gives

$$
\begin{aligned}
& \sum_{\xi \in \text { basis of } V} \hat{\xi} \hat{\triangleleft}\left(\left\langle\xi^{\prime}\right\rangle \triangleleft|\hat{\xi}|\right)^{-1} \otimes \xi^{\prime} \hat{\triangleleft}|\hat{\xi}| \\
& \quad=\sum_{\xi \in \text { basis of } V} \hat{\xi} \hat{\triangleleft}\left(\langle\xi\rangle \triangleleft|\xi|^{-1}\right)^{-1} \otimes T(\xi) \hat{\triangleleft}|\xi|^{-1} .
\end{aligned}
$$

Next,

$$
\begin{aligned}
\theta^{-1}\left(T(\xi) \hat{\triangleleft}|\xi|^{-1}\right) & =\left(T(\xi) \hat{\triangleleft}|\xi|^{-1}\right) \hat{\triangleleft}\left\|T(\xi) \hat{\triangleleft}|\xi|^{-1}\right\|^{-1} \\
& =\left(T(\xi) \hat{\triangleleft}|\xi|^{-1}\right) \hat{\triangleleft}\left(\|T(\xi)\| \tilde{\triangleleft}|\xi|^{-1}\right)^{-1} \\
& =T(\xi) \hat{\triangleleft}|\xi|^{-1}\left(\|\xi\| \tilde{\triangleleft}|\xi|^{-1}\right)^{-1} \\
& =T(\xi) \hat{\triangleleft}|\xi|^{-1}\left(|\xi||\xi|^{-1}\langle\xi\rangle|\xi|^{-1}\right)^{-1} \\
& =T(\xi) \hat{\triangleleft}|\xi|^{-1}|\xi|\langle\xi\rangle^{-1}=T(\xi) \hat{\triangleleft}\langle\xi\rangle^{-1}
\end{aligned}
$$

and finally we need to calculate

$$
\operatorname{eval}\left(\hat{\xi} \triangleleft\left(\langle\xi\rangle \triangleleft|\xi|^{-1}\right)^{-1} \otimes T(\xi) \hat{\triangleleft}\langle\xi\rangle^{-1}\right)=\left(\hat{\xi} \hat{\triangleleft}\left(\langle\xi\rangle \triangleleft|\xi|^{-1}\right)^{-1}\right)\left(T(\xi) \hat{\triangleleft}\langle\xi\rangle^{-1}\right) \text {. }
$$

We know from the definition of the action on $V^{*}$ that

$$
(\hat{\xi} \triangleleft(\|T(\xi)\| \tilde{\triangleright} x))(T(\xi) \hat{\triangleleft} x)=\hat{\xi}(T(\xi)) .
$$


If we put $x=\langle\xi\rangle^{-1}$, we want to show that $\|T(\xi)\| \tilde{\triangleright} x=\left(\langle\xi\rangle \triangleleft|\xi|^{-1}\right)^{-1}$, so

$$
\|\xi\| \tilde{\triangleleft} x=|\xi|^{-1}\langle\xi\rangle \tilde{\triangleleft}\langle\xi\rangle^{-1}=\langle\xi\rangle|\xi|^{-1}=\left(\langle\xi\rangle \triangleright|\xi|^{-1}\right)\left(\langle\xi\rangle \triangleleft|\xi|^{-1}\right)=v^{\prime} t^{\prime},
$$

which implies that $t^{\prime}=\langle\xi\rangle \triangleleft|\xi|^{-1}$, and hence

$$
\begin{aligned}
\|T(\xi)\| \tilde{\triangleright} x & =\|\xi\| \tilde{\triangleright} x=|\xi|^{-1}\langle\xi\rangle \tilde{\triangleright}\langle\xi\rangle^{-1}=t\langle\xi\rangle^{-1} t^{\prime-1} \\
& =\langle\xi\rangle\langle\xi\rangle^{-1}\left(\langle\xi\rangle \triangleleft|\xi|^{-1}\right)^{-1}=\left(\langle\xi\rangle \triangleleft|\xi|^{-1}\right)^{-1} .
\end{aligned}
$$

\section{Characters in the category $\mathscr{D}$}

DEFINITION 7.1 [6]. The right adjoint action in $\mathscr{D}$ of the algebra $D$ on itself is defined by the following diagram:

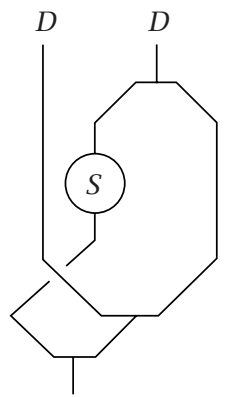

DEFINITION 7.2. The character $\chi_{V}$ of an object $V$ in $\mathscr{D}$ is defined by the following diagram:

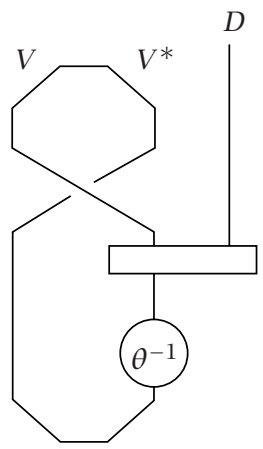

LEMMA 7.3. For an object $V$ in $\mathscr{D}$, the following holds:
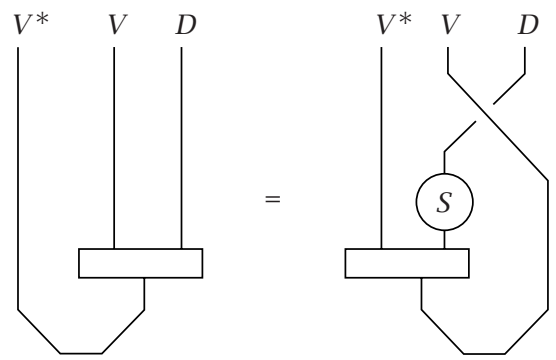


\section{ProOF.}

\section{L.H.S.}
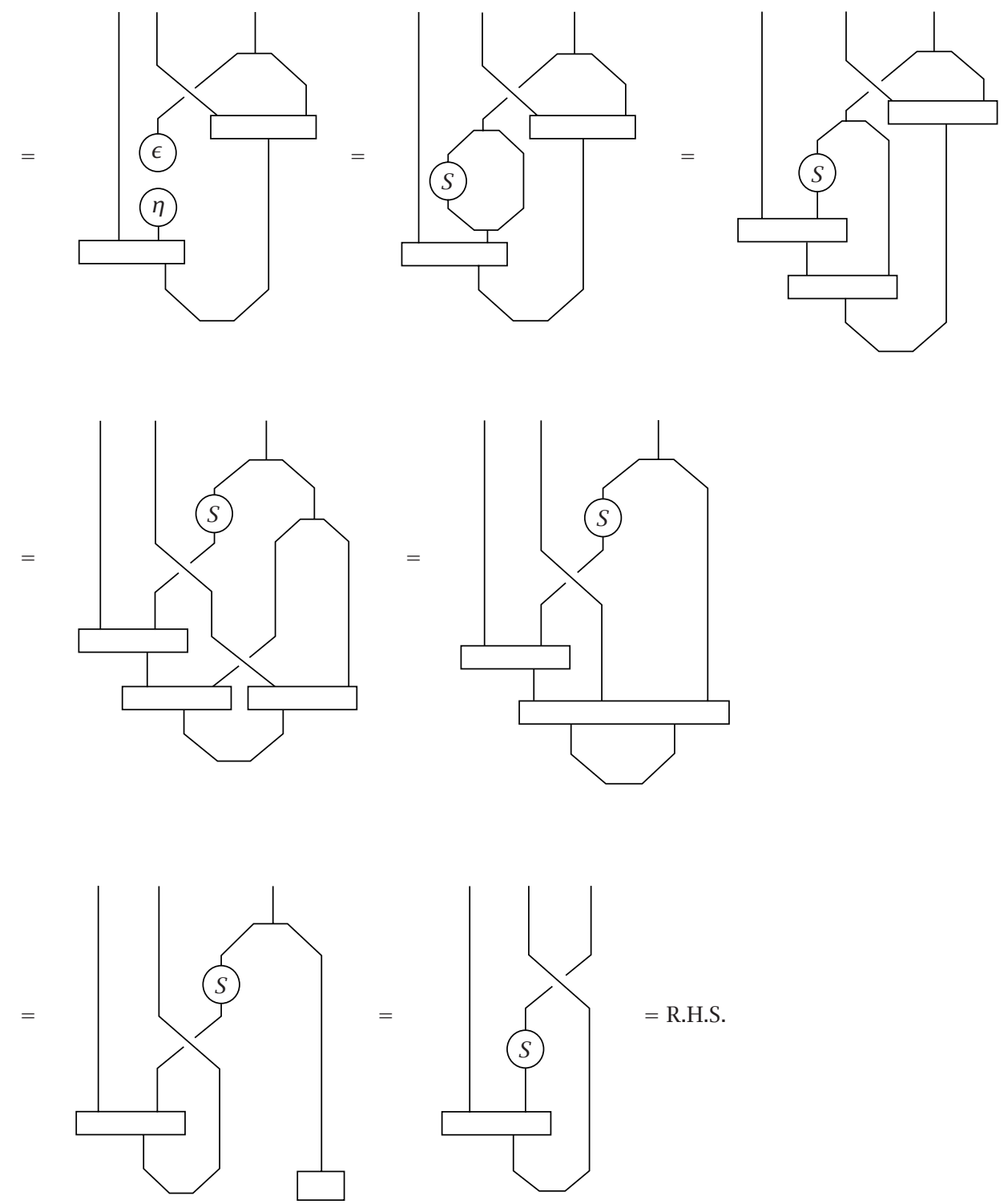
Proposition 7.4. The character is right adjoint invariant, that is, for an object $V$ in $\mathscr{D}$, the following holds:

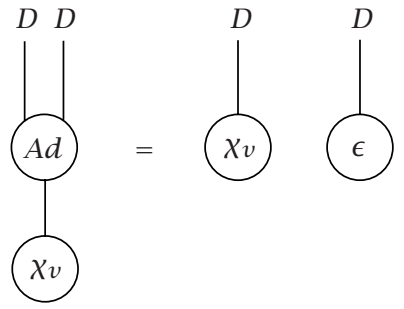

\section{PROOF.}
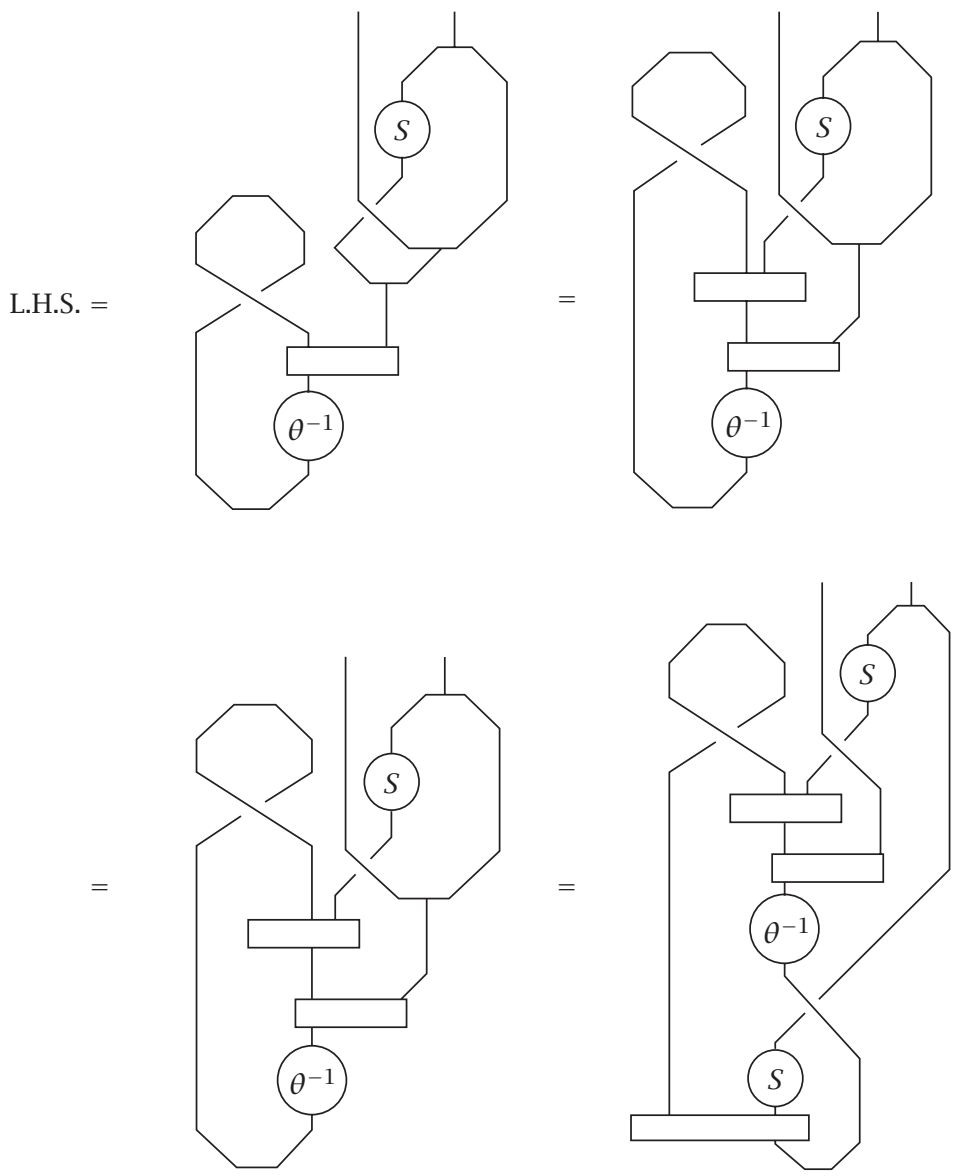

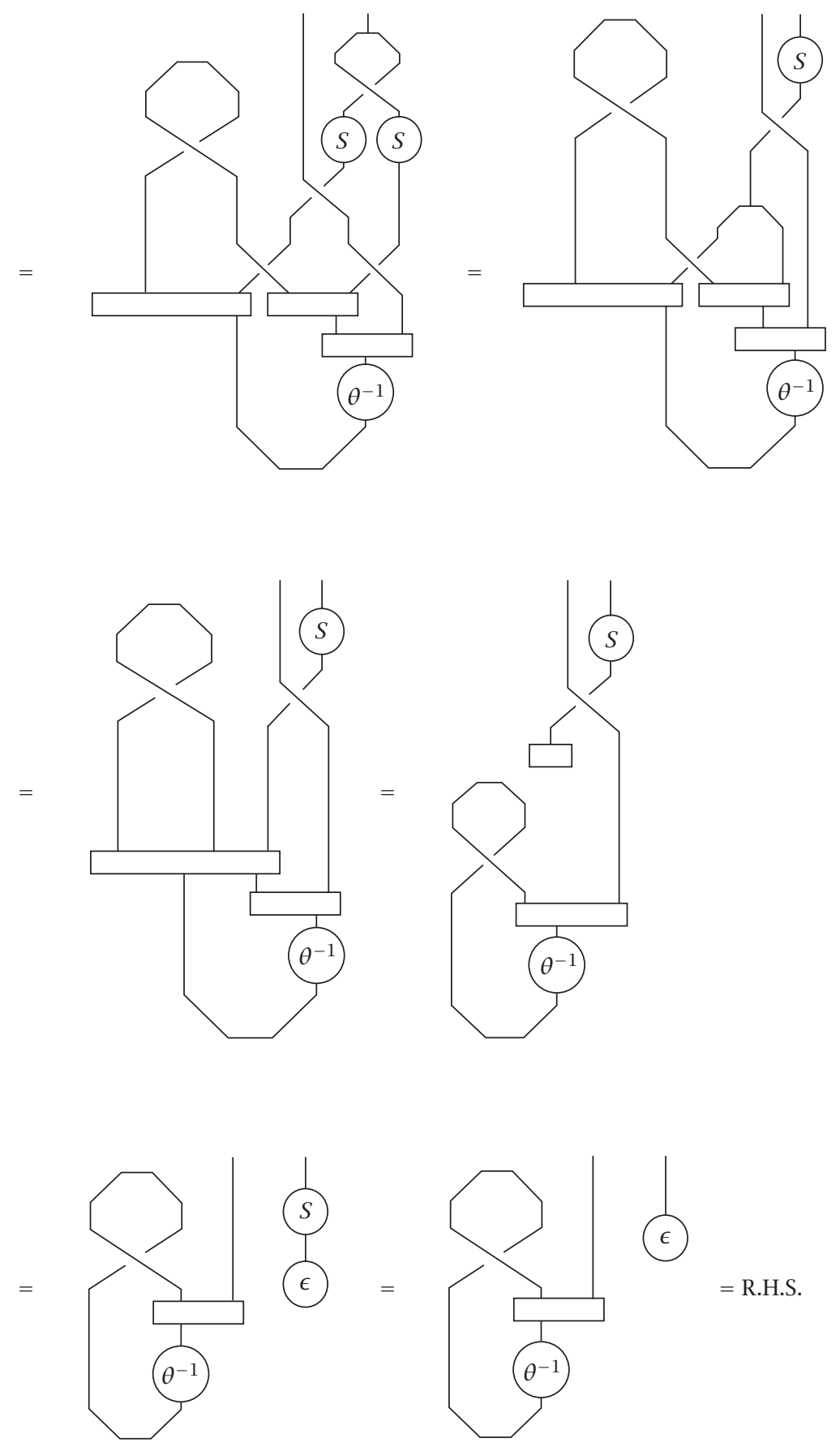
Proposition 7.5. The character of a tensor product of representations is the product of the characters, that is, for two objects $V$ and $W$ in $\mathscr{D}$, the following holds:

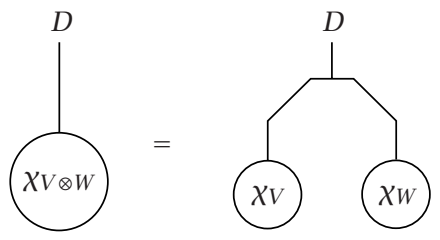

\section{PROOF.}
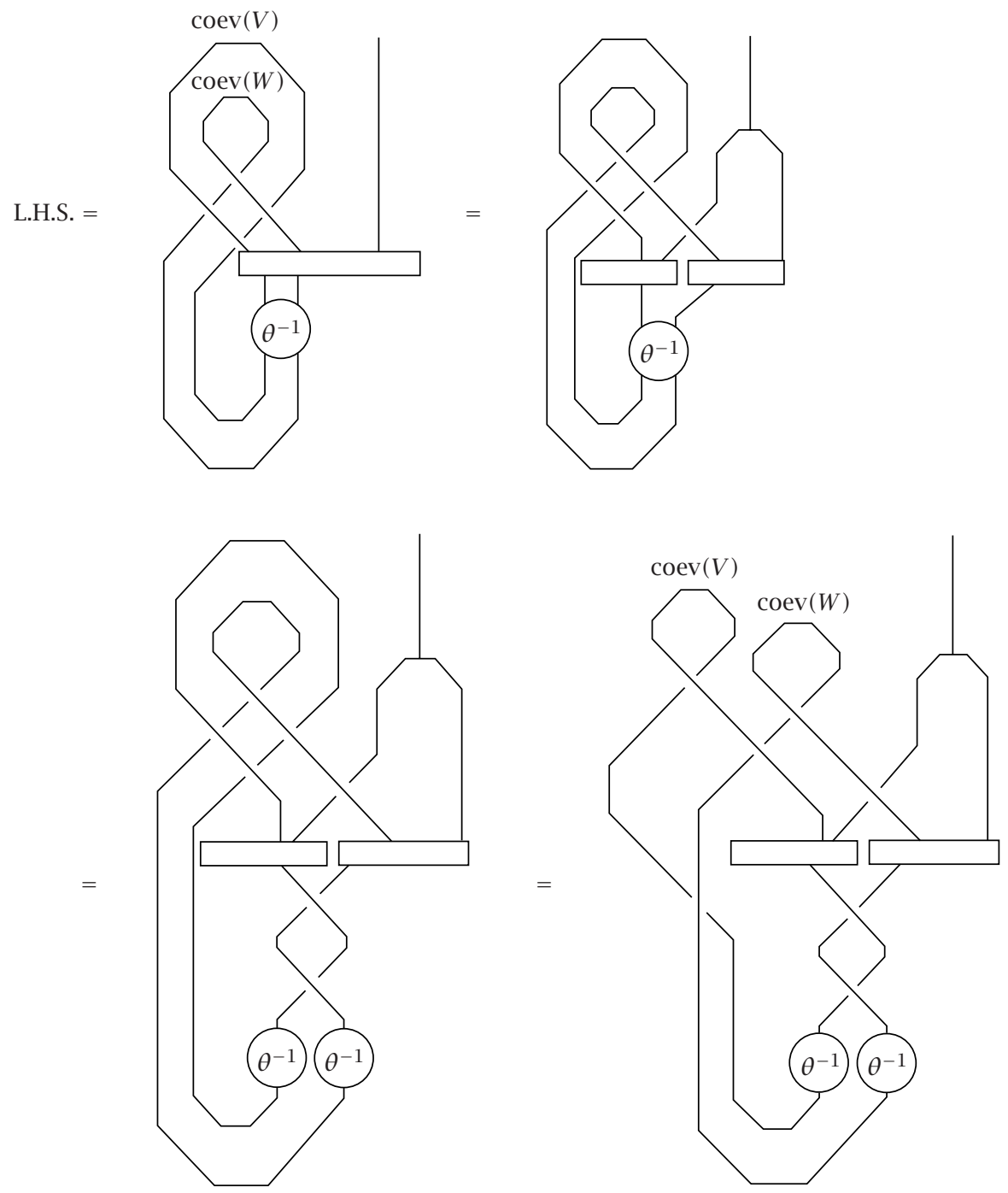

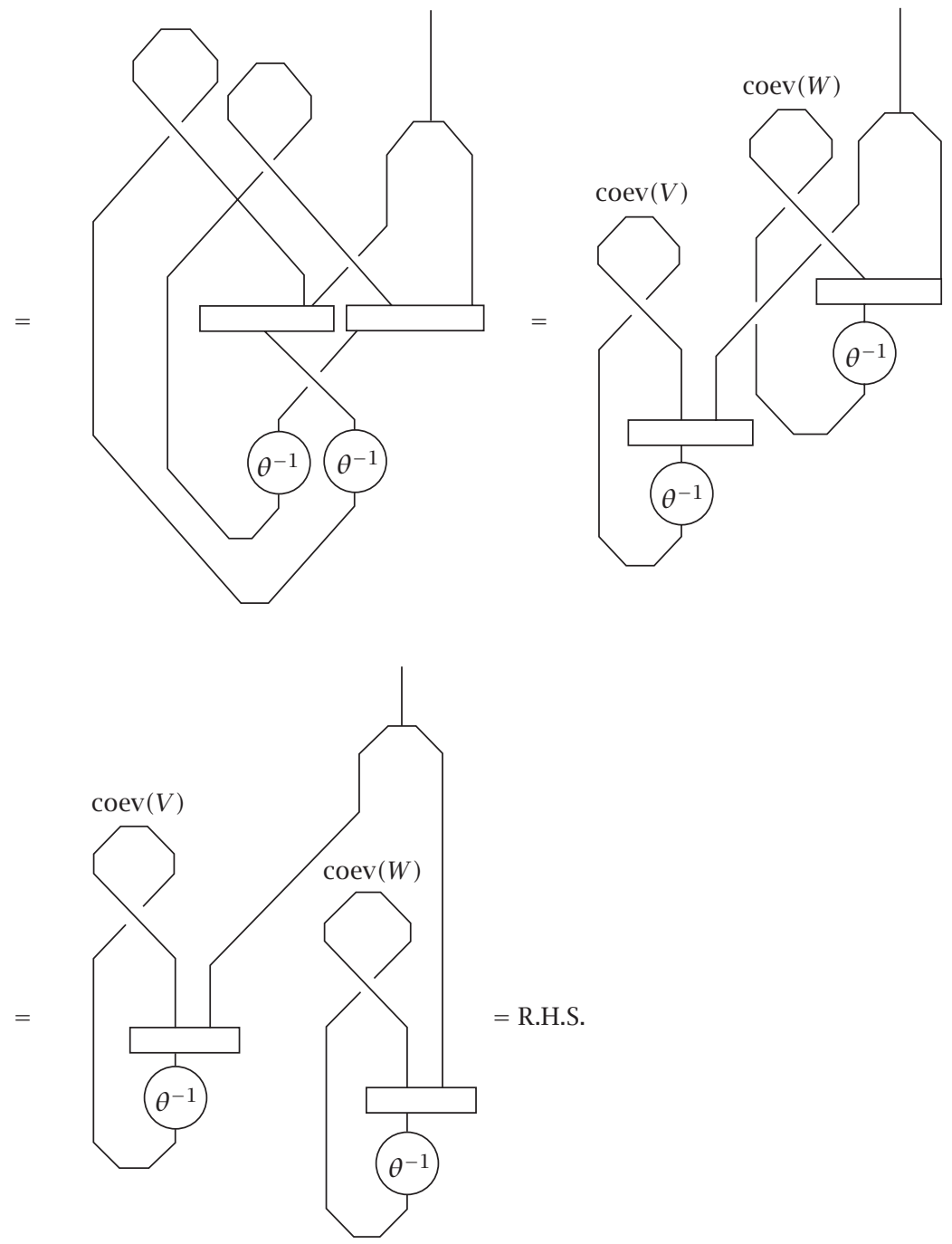

(7.8)

THEOREM 7.6. The following formula holds for the character:

$$
\chi_{V}\left(\delta_{y} \otimes x\right)=\sum_{\xi \in \text { basis of } V, y=\langle\xi\rangle|\xi|^{-1}} \hat{\xi}\left(\xi \hat{\triangleleft}\langle\xi\rangle^{-1} x\langle\xi\rangle\right),
$$

for $x y=y x$, otherwise $\chi_{V}\left(\delta_{y} \otimes x\right)=0$. 
Proof. Set $a=\delta_{y} \otimes x$. To have $\chi_{V}(a) \neq 0$, we must have $\|a\|=e$, that is, $y=y \tilde{\triangleleft} x$, which implies that $x$ and $y$ commute. Assuming this, we continue with the diagrammatic definition of the character, starting with

$$
\left(\sum_{\xi \in \text { basis of } V} \xi \hat{\triangleleft} \tilde{\tau}\left(\|\xi\|^{L},\|\xi\|\right)^{-1} \otimes \hat{\xi}\right) \otimes a=\sum_{\xi \in \text { basis of } V}\left(\xi \hat{\triangleleft} \tau\left(\langle\xi\rangle^{L},\langle\xi\rangle\right)^{-1} \otimes \hat{\xi}\right) \otimes a .
$$

Next, we calculate

$$
\Psi\left(\xi \hat{\triangleleft} \tau\left(\langle\xi\rangle^{L},\langle\xi\rangle\right)^{-1} \otimes \hat{\xi}\right)=\hat{\xi} \hat{\triangleleft}\left(\left\langle\xi^{\prime}\right\rangle \triangleleft|\hat{\xi}|\right)^{-1} \otimes \xi^{\prime} \hat{\triangleleft}|\hat{\xi}|,
$$

where $\xi^{\prime}=\xi \hat{\triangleleft} \tau\left(\langle\xi\rangle^{L},\langle\xi\rangle\right)^{-1}$, so

$$
\left\langle\xi^{\prime}\right\rangle=\left\langle\xi \hat{\triangleleft} \tau\left(\langle\xi\rangle^{L},\langle\xi\rangle\right)^{-1}\right\rangle=\left\langle\xi \triangleleft \tau\left(\langle\xi\rangle^{L},\langle\xi\rangle\right)^{-1}\right\rangle=\langle\xi\rangle \triangleleft \tau\left(\langle\xi\rangle^{L},\langle\xi\rangle\right)^{-1} .
$$

From a previous calculation, we know that $|\hat{\xi}|=\tau\left(\langle\xi\rangle^{L},\langle\xi\rangle\right)|\xi|^{-1}$, so

$$
\begin{aligned}
& \hat{\xi} \triangleleft\left(\left\langle\xi^{\prime}\right\rangle \triangleleft|\hat{\xi}|\right)^{-1}=\hat{\xi} \triangleleft\left(\langle\xi\rangle \triangleleft \tau\left(\langle\xi\rangle^{L},\langle\xi\rangle\right)^{-1} \tau\left(\langle\xi\rangle^{L},\langle\xi\rangle\right)|\xi|^{-1}\right)^{-1} \\
&=\hat{\xi} \triangleleft\left(\langle\xi\rangle \triangleleft|\xi|^{-1}\right)^{-1}, \\
& \xi^{\prime} \triangleleft|\hat{\xi}|=\left(\xi \hat{\triangleleft} \tau\left(\langle\xi\rangle^{L},\langle\xi\rangle\right)^{-1}\right) \hat{\triangleleft}\left(\tau\left(\langle\xi\rangle^{L},\langle\xi\rangle\right)|\xi|^{-1}\right)=\xi \hat{\triangleleft}|\xi|^{-1},
\end{aligned}
$$

which gives the next stage in the evaluation of the diagram:

$$
\begin{aligned}
& \sum_{\xi \in \text { basis of } V} \Psi\left(\xi \hat{\triangleleft} \tau\left(\langle\xi\rangle^{L},\langle\xi\rangle\right)^{-1} \otimes \hat{\xi}\right) \otimes a \\
& \quad=\sum_{\xi \in \text { basis of } V}\left(\hat{\xi} \hat{\triangleleft}\left(\langle\xi\rangle \triangleleft|\xi|^{-1}\right)^{-1} \otimes \xi \hat{\triangleleft}|\xi|^{-1}\right) \otimes a .
\end{aligned}
$$

Now we apply the associator to the last equation to get

$$
\begin{aligned}
& \sum_{\xi \in \text { basis of } V} \Phi\left(\left(\hat{\xi} \hat{\triangleleft}\left(\langle\xi\rangle \triangleleft|\xi|^{-1}\right)^{-1} \otimes \xi \hat{\triangleleft}|\xi|^{-1}\right) \otimes a\right) \\
& =\sum_{\xi \in \text { basis of } V} \hat{\xi} \triangleleft\left(\langle\xi\rangle \triangleleft|\xi|^{-1}\right)^{-1} \tilde{\tau}\left(|| \xi \hat{\triangleleft}|\xi|^{-1}||^{L},\|a\|\right) \otimes\left(\xi \hat{\triangleleft}|\xi|^{-1} \otimes a\right) \\
& =\sum_{\xi \in \text { basis of } V} \hat{\xi} \triangleleft\left(\langle\xi\rangle \triangleleft|\xi|^{-1}\right)^{-1} \tau\left(\left\langle\xi \hat{\triangleleft}|\xi|^{-1}\right\rangle, e\right) \otimes\left(\xi \hat{\triangleleft}|\xi|^{-1} \otimes a\right) \\
& =\sum_{\xi \in \text { basis of } V} \hat{\xi} \triangleleft\left(\langle\xi\rangle \triangleleft|\xi|^{-1}\right)^{-1} \otimes\left(\xi \triangleleft|\xi|^{-1} \otimes\left(\delta_{y} \otimes x\right)\right)
\end{aligned}
$$

as $\tau\left(\left\langle\xi \hat{\triangleleft}|\xi|^{-1}\right\rangle, e\right)=e$. Now apply the action $\hat{\triangleleft}$ to $\xi \hat{\triangleleft}|\xi|^{-1} \otimes\left(\delta_{y} \otimes x\right)$ to get

$$
\left(\xi \hat{\triangleleft}|\xi|^{-1}\right) \hat{\triangleleft}\left(\delta_{y} \otimes x\right)=\delta_{y,\left\|\xi \hat{\jmath}|\xi|^{-1}\right\|}\left(\xi \hat{\triangleleft}|\xi|^{-1}\right) \hat{\triangleleft} x=\delta_{y,\|\xi\| \tilde{\triangleleft}|\xi|^{-1}} \xi \hat{\triangleleft}|\xi|^{-1} x,
$$

and to get a nonzero answer, we must have

$$
y=\|\xi\| \tilde{\triangleleft}|\xi|^{-1}=|\xi|^{-1}\langle\xi\rangle \tilde{\triangleleft}|\xi|^{-1}=|\xi||\xi|^{-1}\langle\xi\rangle|\xi|^{-1}=\langle\xi\rangle|\xi|^{-1} .
$$


Thus the character of $V$ is given by

$$
\chi_{V}\left(\delta_{y} \otimes x\right)=\sum_{\xi \in \text { basis of } V, y=\langle\xi\rangle|\xi|^{-1}} \operatorname{eval}\left(\hat{\xi} \hat{\triangleleft}\left(\langle\xi\rangle \triangleleft|\xi|^{-1}\right)^{-1} \otimes \theta^{-1}\left(\xi \hat{\triangleleft}|\xi|^{-1} x\right)\right) .
$$

Next,

$$
\begin{aligned}
\theta^{-1}\left(\xi \hat{\triangleleft}|\xi|^{-1} x\right) & =\left(\xi \hat{\triangleleft}|\xi|^{-1} x\right) \hat{\triangleleft} \|\left.\xi \hat{\triangleleft}|\xi|^{-1} x\right|^{-1} \\
& =\left(\xi \hat{\triangleleft}|\xi|^{-1} x\right) \hat{\triangleleft}\left(\|\xi\| \tilde{\triangleleft}|\xi|^{-1} x\right)^{-1} \\
& =\left(\xi \hat{\triangleleft}|\xi|^{-1} x\right) \hat{\triangleleft}\left(x^{-1}|\xi||\xi|^{-1}\langle\xi\rangle|\xi|^{-1} x\right)^{-1} \\
& =\xi \hat{\triangleleft}|\xi|^{-1} x x^{-1}|\xi|\langle\xi\rangle^{-1} x=\xi \hat{\triangleleft}\langle\xi\rangle^{-1} x .
\end{aligned}
$$

Now we need to calculate eval $\left(\hat{\xi} \hat{\triangleleft}\left(\langle\xi\rangle \triangleleft|\xi|^{-1}\right)^{-1} \otimes \xi \hat{\triangleleft}\langle\xi\rangle^{-1} x\right)$. Start with $\|\xi\| \tilde{\triangleleft}\langle\xi\rangle^{-1} x=$ $\langle\xi\rangle|\xi|^{-1} \tilde{\triangleleft} x=\langle\xi\rangle|\xi|^{-1}$, as we only have nonzero summands for $y=\langle\xi\rangle|\xi|^{-1}$. Then

$$
\begin{aligned}
\operatorname{eval} & \left(\hat{\xi} \hat{\triangleleft}\left(\langle\xi\rangle \triangleleft|\xi|^{-1}\right)^{-1} \otimes \xi \hat{\triangleleft}\langle\xi\rangle^{-1} x\right) \\
& =\operatorname{eval}\left(\left(\hat{\xi} \hat{\triangleleft}\left(\langle\xi\rangle \triangleleft|\xi|^{-1}\right)^{-1} \otimes \xi \hat{\triangleleft}\langle\xi\rangle^{-1} x\right) \hat{\triangleleft}\langle\xi\rangle\right) \\
& =\operatorname{eval}\left(\hat{\xi} \hat{\triangleleft}\left(\langle\xi\rangle \triangleleft|\xi|^{-1}\right)^{-1}\left(\langle\xi\rangle|\xi|^{-1} \tilde{\triangleright}\langle\xi\rangle\right) \otimes \xi \hat{\triangleleft}\langle\xi\rangle^{-1} x\langle\xi\rangle\right) .
\end{aligned}
$$

To find $\langle\xi\rangle|\xi|^{-1} \tilde{\triangleright}\langle\xi\rangle$, first find $\langle\xi\rangle|\xi|^{-1} \tilde{\triangleleft}\langle\xi\rangle=|\xi|^{-1}\langle\xi\rangle$, so

$$
\begin{aligned}
\langle\xi\rangle|\xi|^{-1} \tilde{\triangleright}\langle\xi\rangle & =\left(\langle\xi\rangle \triangleright|\xi|^{-1}\right)\left(\langle\xi\rangle \triangleleft|\xi|^{-1}\right) \tilde{\triangleright}\langle\xi\rangle \\
& =\left(\langle\xi\rangle \triangleleft|\xi|^{-1}\right)\langle\xi\rangle\langle\xi\rangle^{-1}=\langle\xi\rangle \triangleleft|\xi|^{-1} .
\end{aligned}
$$

LEMmA 7.7. Let $V$ be an object in $\mathscr{D}$. For $\delta_{y} \otimes x \in D$, the character of $V$ is given by the following formula, where $y=s u^{-1}$ with $s \in M$ and $u \in G$ :

$$
\chi_{V}\left(\delta_{y} \otimes x\right)=\sum_{\xi \in \text { basis of } V_{u^{-1} s}} \hat{\xi}\left(\xi \hat{\triangleleft} s^{-1} x s\right)=\chi_{V_{u^{-1}}}\left(s^{-1} x s\right),
$$

where $x y=y x$, otherwise $x_{V}\left(\delta_{y} \otimes x\right)=0$. Here, $x_{V_{u^{-1}}}$ is the group representation character of the representation $V_{u^{-1} s}$ of the group $\operatorname{stab}\left(u^{-1} s\right)$.

Proof. From Theorem 7.6, we know that

$$
\chi_{V}\left(\delta_{y} \otimes x\right)=\sum_{\xi \in \text { basis of } V, y=\langle\xi\rangle|\xi|^{-1}} \hat{\xi}\left(\xi \hat{\triangleleft}\langle\xi\rangle^{-1} x\langle\xi\rangle\right),
$$

for $x y=y x$. Set $s=\langle\xi\rangle$ and $u=|\xi|$, so $y=s u^{-1}$. We note that $s^{-1} x s$ is in $\operatorname{stab}\left(u^{-1} s\right)$, because

$$
u^{-1} s \tilde{\triangleleft} s^{-1} x s=s^{-1} x^{-1} s u^{-1} s s^{-1} x s=s^{-1} x^{-1} x s u^{-1} s=u^{-1} s .
$$

It just remains to note that $\|\xi\|=|\xi|^{-1}\langle\xi\rangle=u^{-1} s$. 
8. Modular categories. Let $\mathcal{M}$ be a semisimple ribbon category. For objects $V$ and $W$ in $\mathcal{M}$, define $\tilde{S}_{V W} \in \underline{\mathbf{1}}$ as follows:

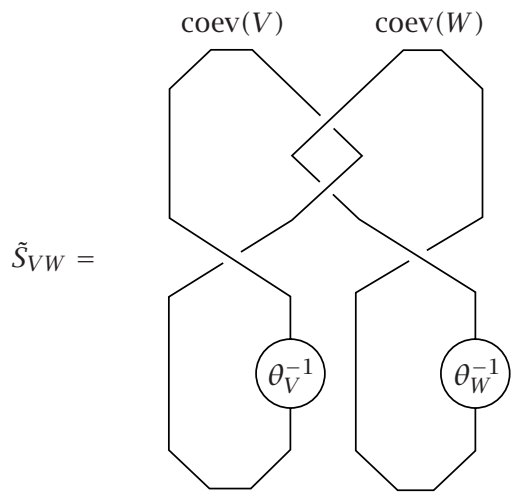

There are standard results [1, 8]:

$$
\tilde{S}_{V W}=\tilde{S}_{W V}=\tilde{S}_{V^{*} W^{*}}=\tilde{S}_{W^{*} V^{*}}, \quad \tilde{S}_{V \underline{1}}=\operatorname{dim}(V) .
$$

Here, $\operatorname{dim}(V)$ is the trace in $\mathcal{M}$ of the identity map on $V$.

Definition 8.1. Call an object $U$ in an abelian category $M$ simple if, for any $V$ in $\mathcal{M}$, any injection $V \hookrightarrow U$ is either 0 or an isomorphism [1]. A semisimple category is an abelian category whose objects split as direct sums of simple objects [8].

DEFINITION 8.2 [1]. A modular category is a semisimple ribbon category $\mathcal{M}$ satisfying the following properties:

(1) there are only a finite number of isomorphism classes of simple objects in $M$,

(2) Schur's lemma holds, that is, the morphisms between simple objects are zero unless they are isomorphic, in which case the morphisms are a multiple of the identity,

(3) the matrix $\tilde{S}_{V W}$ with indices in isomorphism classes of simple objects is invertible.

DEFINITION 8.3 [1]. For a simple object $V$, the ribbon map on $V$ is a multiple of the identity, and $\Theta_{V}$ is used for the scalar multiple. The numbers $P^{ \pm}$are defined as the following sums over simple isomorphism classes:

$$
P^{ \pm}=\sum_{V} \Theta_{V}^{ \pm 1}(\operatorname{dim}(V))^{2}
$$

and the matrices $T$ and $C$ are defined using the Kronecker delta function by

$$
T_{V W}=\delta_{V W} \Theta_{V}, \quad C_{V W}=\delta_{V W^{*}} .
$$


THEOREM 8.4 [1]. In a modular category, if the matrix $S$ is defined by

$$
S=\frac{\tilde{S}}{\sqrt{P^{+} P^{-}}},
$$

then the following matrix equations hold:

$$
(S T)^{3}=\sqrt{\frac{P^{+}}{P^{-}}} S^{2}, \quad S^{2}=C, \quad C T=T C, \quad C^{2}=1 .
$$

We now give some results which allow us to calculate the matrix $\tilde{S}$ in $\mathscr{D}$.

\section{LEMMA 8.5.}

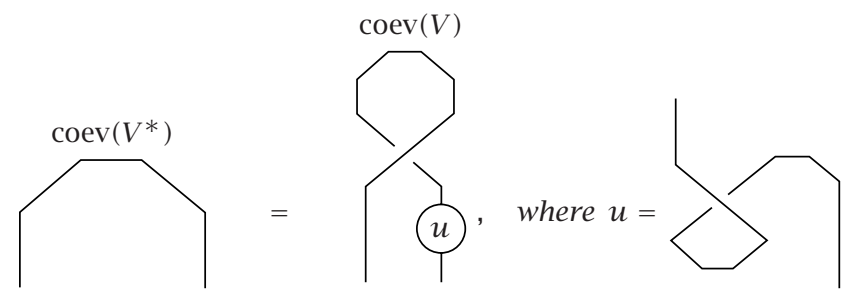

\section{PROOF.}

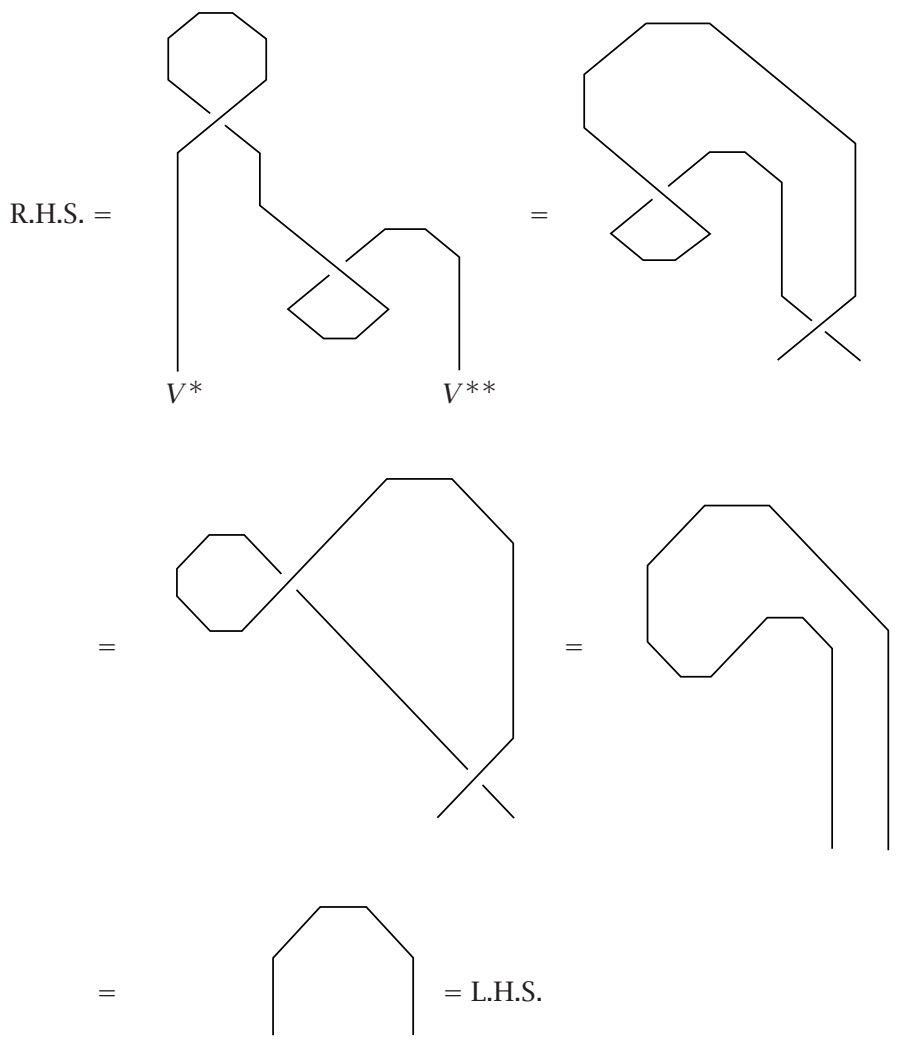




\section{LEMMA 8.6.}
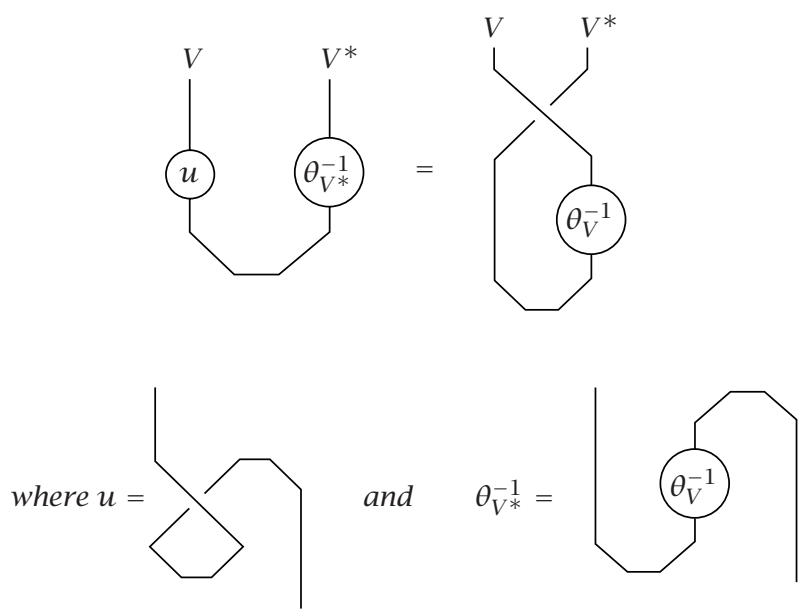

\section{PROOF.}
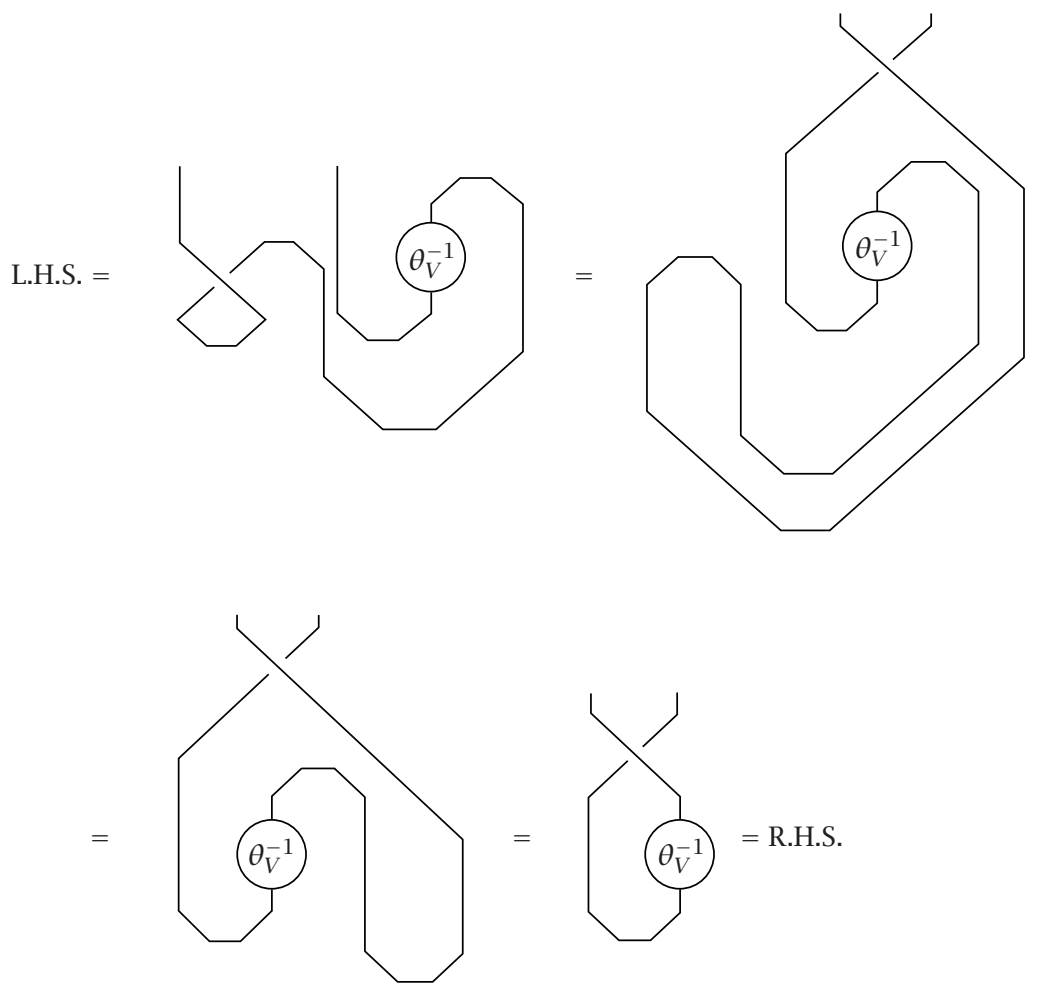

LEMmA 8.7. For $V, W$ indecomposable objects in $\mathscr{D}$, trace $\left(\Psi_{V^{*} W} \circ \Psi_{W V^{*}}\right)=\tilde{S}_{V W}$. 


\section{ProOF.}
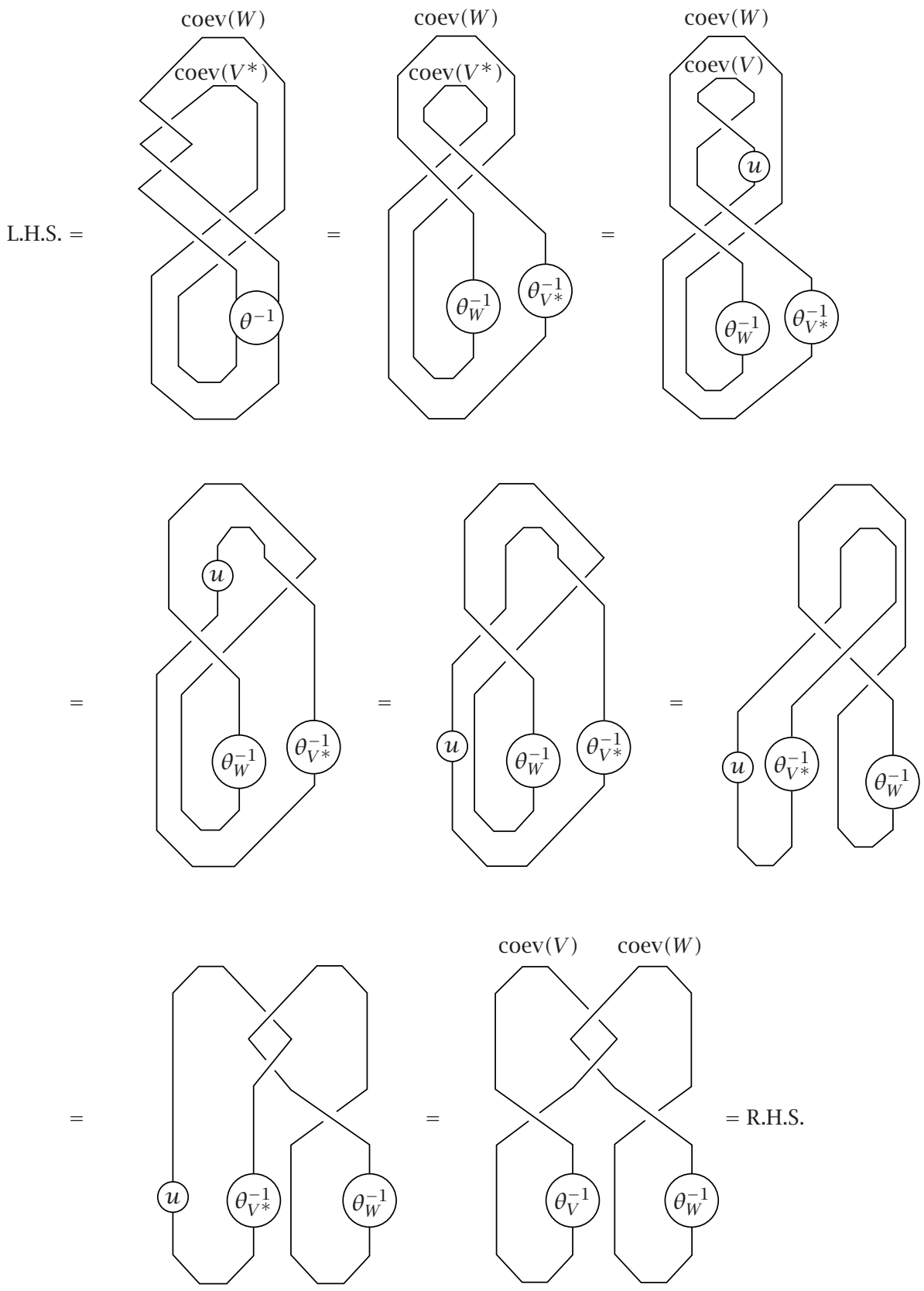
LEMMA 8.8. For two objects $V$ and $W$ in $\mathscr{D}$,

$$
\begin{aligned}
\operatorname{trace}\left(\Psi_{W \otimes V} \circ \Psi_{V \otimes W}\right) \\
=\sum_{\substack{\xi \otimes \eta \in \text { basis of } V \otimes W \\
|\xi|^{-1}\langle\xi\rangle \text { commutes with }|\eta|\langle\eta\rangle^{-1}}} \hat{\eta}\left(\eta \hat{\triangleleft}|\eta|^{-1}\langle\xi\rangle^{-1}|\xi||\eta|\right) \hat{\xi}\left(\xi \hat{\triangleleft}|\eta|\langle\eta\rangle^{-1}\right) .
\end{aligned}
$$

Proof. From Theorem 6.2, we know that

$$
\operatorname{trace}\left(\Psi_{W \otimes V} \circ \Psi_{V \otimes W}\right)=\sum_{(\xi \otimes \eta) \in \text { basis of } V \otimes W}(\widehat{\xi \otimes \eta})\left(\Psi^{2}(\xi \otimes \eta)\right) \text {. }
$$

From the definition of the ribbon map, we know that $\Psi(\Psi(\xi \otimes \eta)) \hat{\triangleleft}\|\xi \otimes \eta\|=\xi \hat{\triangleleft}\|\xi\| \otimes$ $\eta \hat{\triangleleft}\|\eta\|$, so

$$
\begin{aligned}
\Psi(\Psi(\xi \otimes \eta))= & (\xi \hat{\triangleleft}\|\xi\| \otimes \eta \hat{\triangleleft}\|\eta\|) \hat{\triangleleft}\|\xi \otimes \eta\|^{-1} \\
= & \left(\xi \hat{\triangleleft}|\xi|^{-1}\langle\xi\rangle \otimes \eta \hat{\triangleleft}|\eta|^{-1}\langle\eta\rangle\right) \hat{\triangleleft}\langle\eta\rangle^{-1}\langle\xi\rangle^{-1}|\xi||\eta| \\
= & \left(\xi \hat{\triangleleft}|\xi|^{-1}\langle\xi\rangle\right) \hat{\triangleleft}\left(\|\eta \hat{\triangleleft}\| \eta\|\| \tilde{\triangleright}\langle\eta\rangle^{-1}\langle\xi\rangle^{-1}|\xi||\eta|\right) \\
& \otimes \eta \hat{\triangleleft}|\eta|^{-1}\langle\eta\rangle\langle\eta\rangle^{-1}\langle\xi\rangle^{-1}|\xi||\eta| \\
= & \xi \hat{\triangleleft}|\xi|^{-1}\langle\xi\rangle\left(\|\eta\| \tilde{\triangleright}\langle\eta\rangle^{-1}\langle\xi\rangle^{-1}|\xi||\eta|\right) \otimes \eta \hat{\triangleleft}|\eta|^{-1}\langle\xi\rangle^{-1}|\xi \| \eta| .
\end{aligned}
$$

Put $\Psi(\Psi(\xi \otimes \eta))=\xi^{\prime} \otimes \eta^{\prime}$ and $\widehat{\xi \otimes \eta}=\alpha \otimes \beta$, and then from Lemma 4.1 we get

$$
(\widehat{\xi \otimes \eta})\left(\xi^{\prime} \otimes \eta^{\prime}\right)=\left(\alpha \bar{\triangleleft} \tau\left(\langle\beta\rangle,\left\langle\xi^{\prime}\right\rangle \cdot\left\langle\eta^{\prime}\right\rangle\right)\right)\left(\eta^{\prime}\right)\left(\beta \bar{\triangleleft} \tau\left(\left\langle\xi^{\prime}\right\rangle,\left\langle\eta^{\prime}\right\rangle\right)^{-1}\right)\left(\xi^{\prime}\right) .
$$

As $\widehat{\xi \otimes \eta}$ is part of a dual basis, the last expression can only be nonzero if $\left\|\xi^{\prime}\right\|=\|\xi\|$ and $\left\|\eta^{\prime}\right\|=\|\eta\|$. A simple calculation shows that $\left\|\eta^{\prime}\right\|=\|\eta\|$ if and only if $|\xi|^{-1}\langle\xi\rangle$ commutes with $|\eta|\langle\eta\rangle^{-1}$. We use this to find

$$
\begin{aligned}
\|\eta\| \tilde{\triangleleft}\langle\eta\rangle^{-1}\langle\xi\rangle^{-1}|\xi||\eta| & =|\eta|^{-1}|\xi|^{-1}\langle\xi\rangle\langle\eta\rangle|\eta|^{-1}\langle\eta\rangle\langle\eta\rangle^{-1}\langle\xi\rangle^{-1}|\xi||\eta| \\
& =|\eta|^{-1}\langle\eta\rangle|\eta|^{-1}|\xi|^{-1}\langle\xi\rangle\langle\xi\rangle^{-1}|\xi||\eta|=|\eta|^{-1}\langle\eta\rangle,
\end{aligned}
$$

and then

$$
\|\eta\| \tilde{\triangleright}\langle\eta\rangle^{-1}\langle\xi\rangle^{-1}|\xi||\eta|=\langle\eta\rangle\langle\eta\rangle^{-1}\langle\xi\rangle^{-1}|\xi||\eta|\langle\eta\rangle^{-1}=\langle\xi\rangle^{-1}|\xi||\eta|\langle\eta\rangle^{-1}
$$

Now, using the formula for $\widehat{\xi \otimes \eta}=\alpha \otimes \beta$ from Lemma 4.1 gives the result. 
LEMMA 8.9. Let $V$ and $W$ be objects in $\mathscr{D}$. Then in terms of group characters,

$$
\operatorname{trace}\left(\Psi_{V \otimes W}^{2}\right)=\sum_{\substack{u, v \in G, s, t \in M \\ \text { su commutes with vt }}} \chi_{W_{u s}}\left(s^{-1} t^{-1} v^{-1} s\right) \chi_{V_{v t}}\left(u^{-1} s^{-1}\right) \text {. }
$$

Proof. This is more or less immediate from Lemma 8.8. Put $\|\eta\|=u^{-1} s$ and $\|\xi\|=$ $v^{-1} t$ and sum over basis elements of constant degree first.

9. An example of a modular category. Using the order of the indecomposable objects in Table 5.1, we get $T$ to be a diagonal $32 \times 32$ matrix whose diagonal entries are taken from the table. As every indecomposable object in our example is self-dual, the matrix $C$ is the $32 \times 32$ identity matrix.

To find $S$, we calculate the trace of the double braiding trace $\left(\Psi_{V W} \circ \Psi_{W V}\right)$. We do this using the result from Lemma 8.8, split into different cases for the objects $V$ and $W$, and move the points the characters are evaluated at to the base points for each orbit using Lemma 2.3. The following examples are given.

(I) Case (1) $\otimes$ Case (1) (i.e., the orbit of $W$ is $\{e\}$ and the orbit of $V$ is $\{e\}$ ):

$$
\operatorname{trace}\left(\Psi^{2}\right)=\chi_{W_{e}}(e) \chi_{V_{e}}(e) \text {. }
$$

(II) Case (2) $\otimes$ Case (5) (i.e., the orbit of $W$ is $\left\{a^{3}\right\}$ and the orbit of $V$ is $\left\{b, b a^{2}, b a^{4}\right\}$ ):

$$
\operatorname{trace}\left(\Psi^{2}\right)=\left(\chi_{W_{a^{3}}}\left(b a^{2}\right)+\chi_{W_{a^{3}}}\left(b a^{4}\right)+\chi_{W_{a^{3}}}(b)\right) \chi_{V_{b}}\left(a^{3}\right) .
$$

(III) Case (5) $\otimes$ Case (3) (i.e., the orbit of $W$ is $\left\{b, b a^{2}, b a^{4}\right\}$ and the orbit of $V$ is $\left\{a^{2}, a^{4}\right\}$ ):

$$
\operatorname{trace}\left(\Psi^{2}\right)=0
$$

(IV) Case (6) $\otimes$ Case (5) (i.e., the orbit of $W$ is $\left\{b a, b a^{3}, b a^{5}\right\}$ and the orbit of $V$ is $\left\{b, b a^{2}\right.$, $\left.\left.b a^{4}\right\}\right)$ :

$$
\operatorname{trace}\left(\Psi^{2}\right)=3\left(\chi_{W_{b a}}\left(b a^{4}\right) \chi_{V_{b}}\left(b a^{3}\right)\right)
$$

Noting that the dimension in $D$ of each $V$ is the same as its usual dimension, we get $P^{+}=P^{-}=12$. 
From these cases, we get $S$ to be one twelfth of the following $32 \times 32$ symmetric matrix:

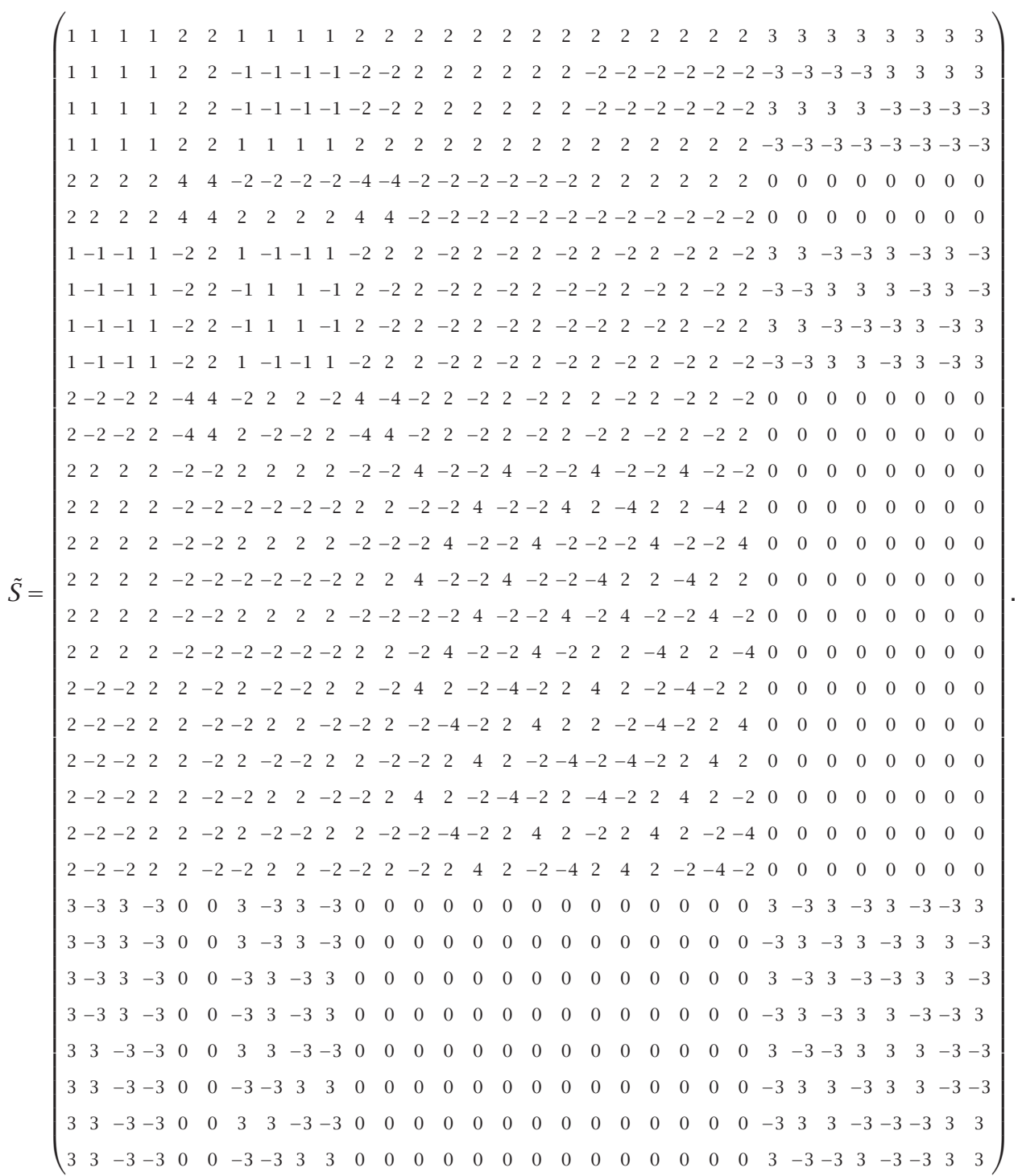

Now it is possible to check that the matrices $S, T$, and $C$ satisfy the following relations:

$$
S^{2}=(S T)^{3}, \quad C S=S C, \quad C T=T C .
$$

10. An equivalence of tensor categories. In this section, we will generalize some results of [3] which considered group double cross products, that is, a group $X$ factoring into two subgroups $G$ and $M$. 
DEFINITION 10.1 [3]. For the double cross product group $X=G M$, there is a quantum double $D(X)=k(X) \rtimes k X$ which has the following operations:

$$
\begin{aligned}
& \left(\delta_{y} \otimes x\right)\left(\delta_{y^{\prime}} \otimes x^{\prime}\right)=\delta_{x^{-1} y x, y^{\prime}}\left(\delta_{y} \otimes x x^{\prime}\right), \quad \Delta\left(\delta_{y} \otimes x\right)=\sum_{a b=y} \delta_{a} \otimes x \otimes \delta_{b} \otimes x, \\
& 1=\sum_{y} \delta_{y} \otimes e, \quad \epsilon\left(\delta_{y} \otimes x\right)=\delta_{y, e}, \quad S\left(\delta_{y} \otimes x\right)=\delta_{x^{-1} y^{-1} x} \otimes x^{-1}, \\
& \left(\delta_{y} \otimes x\right)^{*}=\delta_{x^{-1} y x} \otimes x^{-1}, \quad R=\sum_{x, z} \delta_{x} \otimes e \otimes \delta_{z} \otimes x .
\end{aligned}
$$

The representations of $D(X)$ are given by $X$-graded left $k X$-modules. The $k X$-action will be denoted by $\dot{\triangleright}$ and the grading by \|\|$\cdot \|$. The grading and $X$-action are related by

$$
\left\|\left|x \dot { \triangleright } \xi \left\||=x \|||\xi| \mid x^{-1}, \quad x \in X, \xi \in V,\right.\right.\right.
$$

and the action of $\left(\delta_{y} \otimes x\right) \in D(X)$ is given by

$$
\left(\delta_{y} \otimes x\right) \dot{\triangleright} \xi=\delta_{y,\|\| x \dot{\triangleright} \xi \|} x \dot{\triangleright} \xi .
$$

Proposition 10.2. There is a functor $x$ from $\mathscr{D}$ to the category of representations of $D(X)$ given by the following: as vector spaces, $\chi(V)$ is the same as $V$, and $\chi$ is the identity map. The $X$-grading $|\|\cdot\||$ on $\chi(V)$ and the action of us $\in k X$ are defined by

$$
\begin{gathered}
|||\chi(\eta)|||=\langle\eta\rangle^{-1}|\eta| \quad \text { for } \eta \in V, \\
u s \dot{\triangleright} \chi(\eta)=\chi\left(\left(\left(s \triangleleft|\eta|^{-1}\right) \triangleright \bar{\triangleright}\right) \triangleleft u^{-1}\right), \quad s \in M, u \in G .
\end{gathered}
$$

A morphism $\phi: V \rightarrow W$ in $\mathscr{D}$ is sent to the morphism $\chi(\phi): \chi(V) \rightarrow \chi(W)$ defined by $\chi(\phi)(\chi(\xi))=\chi(\phi(\xi))$.

Proof. First, we show that $\dot{\triangleright}$ is an action, that is, $v t \dot{\triangleright}(u s \dot{\triangleright} \chi(\eta))=v t u s \dot{\triangleright} \chi(\eta)$ for all $s, t \in M$ and $u, v \in G$. Note that

$$
\begin{aligned}
v t \dot{\triangleright}(u s \dot{\triangleright} \chi(\eta)) & =v t \dot{\triangleright} \chi\left(\left(\left(s \triangleleft|\eta|^{-1}\right) \bar{\triangleright} \eta\right) \bar{\triangleleft} u^{-1}\right) \\
& =\chi\left(\left(\left(t \triangleleft|\bar{\eta}|^{-1}\right) \bar{\triangleright} \bar{\eta}\right) \bar{\triangleleft} v^{-1}\right),
\end{aligned}
$$

where $\bar{\eta}=\left(\left(s \triangleleft|\eta|^{-1}\right) \bar{\triangleright} \eta\right) \bar{\triangleleft} u^{-1}$. On the other hand, we have

$$
v t u s=v(t \triangleright u) \tau(t \triangleleft u, s)((t \triangleleft u) \cdot s),
$$

where $v(t \triangleright u) \tau(t \triangleleft u, s) \in G$ and $(t \triangleleft u) \cdot s \in M$, so

$$
\left.v t u s \dot{\triangleright} \chi(\eta)=\chi\left(\left(((t \triangleleft u) \cdot s) \triangleleft|\eta|^{-1}\right) \bar{\triangleright} \eta\right) \bar{\triangleleft} \tau(t \triangleleft u, s)^{-1}(t \triangleright u)^{-1} v^{-1}\right) .
$$

We need to show that

$$
\begin{aligned}
\left(t \triangleleft|\bar{\eta}|^{-1}\right) \bar{\triangleright} \bar{\eta} & =\left(\left(((t \triangleleft u) \cdot s) \triangleleft|\eta|^{-1}\right) \bar{\triangleright} \eta\right) \bar{\triangleleft} \tau(t \triangleleft u, s)^{-1}(t \triangleright u)^{-1} \\
& =\left(\left(\left(t \triangleleft u\left(s \triangleright|\eta|^{-1}\right)\right) \cdot\left(s \triangleleft|\eta|^{-1}\right)\right) \bar{\triangleright} \eta\right) \bar{\triangleleft} \tau(t \triangleleft u, s)^{-1}(t \triangleright u)^{-1} .
\end{aligned}
$$


Put $\bar{s}=s \triangleleft|\eta|^{-1}$ and $\eta^{\prime}=\bar{s} \bar{\triangleright} \eta$ which give $\bar{\eta}=\eta^{\prime} \triangleleft u^{-1}$. Then, using the connections between the gradings and actions,

$$
|\bar{\eta}|=\left|\eta^{\prime} \triangleleft u^{-1}\right|=\left(\left\langle\eta^{\prime}\right\rangle \triangleright u^{-1}\right)^{-1}\left|\eta^{\prime}\right| u^{-1} .
$$

Putting $\bar{t}=t \triangleleft u\left|\eta^{\prime}\right|^{-1}$, the left-hand side of (10.8) will become

$$
\begin{aligned}
\left(t \triangleleft|\bar{\eta}|^{-1}\right) \triangleright \bar{\eta} & =\left(t \triangleleft u\left|\eta^{\prime}\right|^{-1}\left(\left\langle\eta^{\prime}\right\rangle \triangleright u^{-1}\right)\right) \bar{\triangleright}\left(\eta^{\prime} \triangleleft u^{-1}\right) \\
& =\left(\bar{t} \triangleleft\left(\left\langle\eta^{\prime}\right\rangle \triangleright u^{-1}\right)\right) \triangleright\left(\eta^{\prime} \triangleleft u^{-1}\right) \\
& =\left(\bar{t} \bar{\triangleright} \eta^{\prime}\right) \bar{\triangleleft}\left(\left(\bar{t} \triangleleft\left|\eta^{\prime}\right|\right) \triangleright u^{-1}\right) .
\end{aligned}
$$

Now, from (10.8) and the fact that $(t \triangleright u)^{-1}=\left(\bar{t} \triangleleft\left|\eta^{\prime}\right|\right) \triangleright u^{-1}$, we only need to show that

$$
\bar{t} \bar{\triangleright} \eta^{\prime}=\left(\left(\left(t \triangleleft u\left(s \triangleright|\eta|^{-1}\right)\right) \cdot\left(s \triangleleft|\eta|^{-1}\right)\right) \bar{\triangleright} \eta\right) \bar{\triangleleft} \tau(t \triangleleft u, s)^{-1} .
$$

From the formula for the composition of the $M$ "action," the right-hand side of (10.11) becomes $\bar{p} \bar{\triangleright}(\bar{s} \bar{\triangleright} \eta)=\bar{p} \bar{\triangleright} \eta^{\prime}$, where $\bar{p}^{\prime}=t \triangleleft u\left(s \triangleright|\eta|^{-1}\right)$ and $\bar{p}=\bar{p}^{\prime} \triangleleft \tau(\bar{s},\langle\eta\rangle) \tau(\langle\bar{s} \bar{\triangleright} \eta\rangle$, $\bar{s} \triangleleft|\eta|)^{-1}$. We have used the fact that $\tau(t \triangleleft u, s)=\tau\left(\bar{p}^{\prime} \triangleleft(\bar{s} \triangleright|\eta|), \bar{s} \triangleleft|\eta|\right)$. Now we just have to prove that $\bar{p}=\bar{t}$. Because $\tau(\bar{s},\langle\eta\rangle)^{-1}(\bar{s} \triangleright|\eta|)=\tau(\langle\bar{s} \bar{\triangleright} \eta\rangle, \bar{s} \triangleleft|\eta|)^{-1}|\bar{s} \bar{\triangleright} \eta|$ and knowing that $(\bar{s} \triangleright|\eta|)=\left(s \triangleright|\eta|^{-1}\right)^{-1}$, we can write $\bar{p}$ as follows:

$$
\begin{aligned}
\bar{p} & =\bar{p}^{\prime} \triangleleft(\bar{s} \triangleright|\eta|)|\bar{s} \triangleright \eta|^{-1} \\
& =t \triangleleft u\left(s \triangleright|\eta|^{-1}\right)\left(s \triangleright|\eta|^{-1}\right)^{-1}\left|\eta^{\prime}\right|^{-1} \\
& =t \triangleleft u\left|\eta^{\prime}\right|^{-1}=\bar{t} .
\end{aligned}
$$

Next, we show that \|\|$u s \dot{\triangleright} \chi(\eta)\|\|=u s|\|\chi(\eta)\||(u s)^{-1}$, where $u \in G$ and $s \in M$ :

$$
\begin{aligned}
\|x \dot{\triangleright} \chi(\eta)\| & =\|\left|x\left(\left(\left(s \triangleleft|\eta|^{-1}\right) \bar{\triangleright} \eta\right) \bar{\triangleleft} u^{-1}\right)\right||| \\
& =\left\langle\eta^{\prime} \bar{\triangleleft} u^{-1}\right\rangle^{-1}\left|\eta^{\prime} \bar{\triangleleft} u^{-1}\right| \\
& =u\left\langle\eta^{\prime}\right\rangle^{-1}\left|\eta^{\prime}\right| u^{-1} \\
& =u\langle\bar{s} \bar{\triangleright} \eta\rangle^{-1}|\bar{s} \bar{\triangleright} \eta| u^{-1} \\
& =u(\bar{s} \triangleleft|\eta|)\langle\eta\rangle^{-1}|\eta|(\bar{s} \triangleleft|\eta|)^{-1} u^{-1} \\
& =u s\langle\eta\rangle^{-1}|\eta| s^{-1} u^{-1} .
\end{aligned}
$$

THEOREM 10.3. The functor $x$ is invertible.

Proof. We have already proved in Proposition 10.2 that the $X$-grading $\||\cdot|\|$ and the action $\dot{\triangleright}$ give a representation of $D(X)$, so we only need to show that $\chi$ is a oneto-one correspondence, which we do by giving its inverse $\chi^{-1}$ as follows: let $W$ be a representation of $D(X)$, with $k X$-action $\dot{\triangleright}$ and $X$-grading $\||\cdot|\|$. Define a $D$ representation as follows: $X^{-1}(W)$ will be the same as $W$ as a vector space. There will be $G$ - and $M$-gradings given by the factorization

$$
\left\|\left.\left|\xi \|^{-1}=\right| \chi^{-1}(\xi)\right|^{-1}\left\langle\chi^{-1}(\xi)\right\rangle, \quad \xi \in W,\left\langle\chi^{-1}(\xi)\right\rangle \in M,\left|\chi^{-1}(\xi)\right| \in G .\right.
$$


The actions of $s \in M$ and $u \in G$ are given by

$$
s \bar{\triangleright} \chi^{-1}(\xi)=\chi^{-1}\left(\left(s \triangleleft\left|\chi^{-1}(\xi)\right|\right) \dot{\triangleright} \xi\right), \quad \chi^{-1}(\xi) \triangleleft u=\chi^{-1}\left(u^{-1} \dot{\triangleright} \xi\right) .
$$

Checking the rest is left to the reader.

Proposition 10.4. For $\delta_{y} \otimes x \in \mathscr{D}, \chi\left(\xi \hat{\triangleleft}\left(\delta_{y} \otimes x\right)\right)=\delta_{y,\|\xi\|} x^{-1} \dot{\triangleright} \chi(\xi)$.

Proof. Starting with the left-hand side,

$$
\chi\left(\xi \hat{\triangleleft}\left(\delta_{y} \otimes x\right)\right)=\chi\left(\delta_{y,\|\xi\|} \xi \hat{\triangleleft} x\right)=\delta_{y,\|\xi\| \chi}(\xi \hat{\triangleleft} x) .
$$

Putting $x=u s$ for $u \in G$ and $s \in M$,

$$
\xi \hat{\triangleleft} x=\xi \hat{\triangleleft} u s=(\xi \triangleleft u) \hat{\triangleleft} s=\left(\left(s^{L} \triangleleft u^{-1}|\xi|^{-1}\right) \bar{\triangleright} \xi\right) \triangleleft\left(s^{L} \triangleright u^{-1}\right)^{-1} \tau\left(s^{L}, s\right) .
$$

Now put $\bar{u}=\tau\left(s^{L}, s\right)^{-1}\left(s^{L} \triangleright u^{-1}\right)$ and $\bar{s}=s^{L} \triangleleft u^{-1}$. Then

$$
\begin{aligned}
& \chi\left(\xi \hat{\triangleleft}\left(\delta_{y} \otimes x\right)\right)=\delta_{y,\|\xi\| \chi}\left(\left(\left(\bar{s} \triangleleft|\xi|^{-1}\right) \bar{\triangleright} \xi\right) \bar{\triangleleft} \bar{u}^{-1}\right)=\delta_{y,\|\xi\|} \bar{u} \bar{s} \dot{\triangleright} \chi(\xi) \\
& =\delta_{y,\|\xi\|} \boldsymbol{T}\left(s^{L}, s\right)^{-1}\left(s^{L} \triangleright u^{-1}\right)\left(s^{L} \triangleleft u^{-1}\right) \dot{\triangleright} \chi(\xi) \\
& =\delta_{y,\|\xi\| s^{-1} s^{L^{-1}} s^{L} u^{-1} \dot{\triangleright} \chi(\xi)} \\
& =\delta_{y,\|\xi\|}(u s)^{-1} \dot{\triangleright} \chi(\xi) \\
& =\delta_{y,\|\xi\|} x^{-1} \dot{\triangleright} \chi(\xi) \text {. }
\end{aligned}
$$

Proposition 10.5. Define a map $\psi: D \rightarrow D(X)$ by $\psi\left(\delta_{y} \otimes x\right)=\delta_{x^{-1} y x} \otimes x^{-1}$. Then $\psi$ satisfies the equation $\chi\left(\xi \grave{\triangleleft}\left(\delta_{y} \otimes x\right)\right)=\psi\left(\delta_{y} \otimes x\right) \dot{\triangleright} \chi(\xi)$.

Proof. Use the previous proposition.

The reader will recall that $D$ is in general a nontrivially associated algebra (i.e., it is only associative in the category $\mathscr{D}$ with its nontrivial associator). Thus, in general, it cannot be isomorphic to $D(X)$, which is really associative. In general, $\psi$ cannot be an algebra map.

Proposition 10.6. For $a$ and $b$ elements of the algebra $D$ in the category $\mathscr{D}$,

$$
\psi(b) \psi(a)=\psi(a b)\left(\sum_{y \in Y} \delta_{y} \otimes \tau(\langle a\rangle,\langle b\rangle)^{-1}\right) .
$$

Proof. By Proposition 10.5, we have

$$
\begin{aligned}
\chi((\xi \hat{\triangleleft} a) \hat{\triangleleft} b) & =\psi(b) \dot{\triangleright} \chi(\xi \hat{\triangleleft} a)=\psi(b) \dot{\triangleright}(\psi(a) \dot{\triangleright} \chi(\xi)) \\
& =\psi(b) \psi(a) \dot{\triangleright} \chi(\xi) .
\end{aligned}
$$

But also, where $f=\sum_{y} \delta_{y} \otimes \tau(\langle a\rangle,\langle b\rangle)$,

$$
\begin{aligned}
\chi((\xi \hat{\triangleleft} a) \hat{\triangleleft} b) & =\chi((\xi \hat{\triangleleft} \tilde{\tau}(\|a\|,\|b\|)) \hat{\triangleleft} a b)=\psi(a b) \dot{\triangleright} \chi(\xi \hat{\triangleleft} \tilde{\tau}(\|a\|,\|b\|)) \\
& =\psi(a b) \dot{\triangleright} \chi(\xi \hat{\triangleleft} \tilde{\tau}(\langle a\rangle,\langle b\rangle))=\psi(a b) \psi(f) \dot{\triangleright} \chi(\xi) .
\end{aligned}
$$


DEFINITION 10.7. Let $V$ and $W$ be objects of $\mathscr{D}$. The map $c: \chi(V) \otimes \chi(W) \rightarrow \chi(V \otimes W)$ is defined by

$$
c(\chi(\eta) \otimes \chi(\xi))=\chi\left(\left(\left(\langle\xi\rangle \triangleleft|\eta|^{-1}\right) \bar{\triangleright} \eta\right) \otimes \xi\right) .
$$

Proposition 10.8. The map c, defined above, is a $D(X)$ module map, that is,

$$
\begin{gathered}
\||c(\chi(\eta) \otimes \chi(\xi))\|=\mid\| \chi(\eta) \otimes \chi(\xi)\|\|, \\
x \dot{\triangleright} c(\chi(\eta) \otimes \chi(\xi))=c(x \dot{\triangleright}(\chi(\eta) \otimes \chi(\xi))) \quad \forall x \in X .
\end{gathered}
$$

Proof. We will begin with the grading first. It is known that

$$
\||x(\eta) \otimes \chi(\xi)|||=|||\chi(\eta)||||||\chi(\xi)|||=\langle\eta\rangle^{-1}|\eta|\langle\xi\rangle^{-1}|\xi| .
$$

But, on the other hand, we know from the definition of $c$ that

$$
\begin{aligned}
\|c(\chi(\eta) \otimes \chi(\xi))\| & =\||| \chi\left(\left(\langle\xi\rangle \triangleleft|\eta|^{-1}\right) \bar{\triangleright} \eta \otimes \xi\right)|| \mid \\
& =\left\langle\left(\langle\xi\rangle \triangleleft|\eta|^{-1}\right) \bar{\triangleright} \eta \otimes \xi\right\rangle^{-1}\left|\left(\langle\xi\rangle \triangleleft|\eta|^{-1}\right) \bar{\triangleright} \eta \otimes \xi\right| \\
& =\langle\xi\rangle^{-1}\langle\bar{\eta}\rangle^{-1}|\bar{\eta}||\xi| \\
& =\langle\xi\rangle^{-1}\langle\bar{s} \bar{\triangleright} \eta\rangle^{-1}|\bar{s} \bar{\triangleright} \eta||\xi| \\
& =\langle\xi\rangle^{-1}(\bar{s} \triangleleft|\eta|)\langle\eta\rangle^{-1}|\eta|(\bar{s} \triangleleft|\eta|)^{-1}|\xi| \\
& =\langle\eta\rangle^{-1}|\eta|\langle\xi\rangle^{-1}|\xi|,
\end{aligned}
$$

where $\bar{s}=\langle\xi\rangle \triangleleft|\eta|^{-1}$ and $\bar{\eta}=\left(\langle\xi\rangle \triangleleft|\eta|^{-1}\right) \bar{\nabla} \eta=\bar{s} \triangleright \eta$, which gives the result.

For the $G$-action, we know from the definitions that

$$
\begin{aligned}
u \dot{\triangleright}(\chi(\eta) \otimes \chi(\xi)) & =\chi\left(\eta \bar{\triangleleft} u^{-1}\right) \otimes \chi\left(\xi \bar{\triangleleft} u^{-1}\right), \\
c(u \dot{\triangleright}(\chi(\eta) \otimes \chi(\xi))) & =\chi\left(\left(\left(\left\langle\xi \bar{\triangleleft} u^{-1}\right\rangle \triangleleft\left|\eta \bar{\triangleleft} u^{-1}\right|^{-1}\right) \bar{\triangleright}\left(\eta \bar{\triangleleft} u^{-1}\right)\right) \otimes\left(\xi \bar{\triangleleft} u^{-1}\right)\right) .
\end{aligned}
$$

By using the properties of the $G$ - and $M$-gradings,

$$
\begin{aligned}
\left\langle\xi \bar{\triangleleft} u^{-1}\right\rangle \triangleleft\left|\eta \bar{\triangleleft} u^{-1}\right|^{-1} & =\left(\langle\xi\rangle \triangleleft u^{-1}\right) \triangleleft u|\eta|^{-1}\left(\langle\eta\rangle \triangleright u^{-1}\right) \\
& =\langle\xi\rangle \triangleleft|\eta|^{-1}\left(\langle\eta\rangle \triangleright u^{-1}\right), \\
\left(\left\langle\xi \bar{\triangleleft} u^{-1}\right\rangle \triangleleft\left|\eta \bar{\triangleleft} u^{-1}\right|^{-1}\right) \bar{\triangleright}\left(\eta \bar{\triangleleft} u^{-1}\right) & =\left(\left(\langle\xi\rangle \triangleleft|\eta|^{-1}\right) \triangleleft\left(\langle\eta\rangle \triangleright u^{-1}\right)\right) \triangleright\left(\eta \bar{\triangleleft} u^{-1}\right) \\
& =\left(\left(\langle\xi\rangle \triangleleft|\eta|^{-1}\right) \triangleright \eta\right) \bar{\triangleleft}\left(\left(\left(\langle\xi\rangle \triangleleft|\eta|^{-1}\right) \triangleleft|\eta|\right) \triangleright u^{-1}\right) \\
& =\left(\left(\langle\xi\rangle \triangleleft|\eta|^{-1}\right) \triangleright \eta\right) \triangleleft\left(\langle\xi\rangle \triangleright u^{-1}\right) .
\end{aligned}
$$

Now we can write

$$
c(u \dot{\triangleright}(\chi(\eta) \otimes \chi(\xi)))=\chi\left(\left(\left(\langle\xi\rangle \triangleleft|\eta|^{-1}\right) \bar{\triangleright} \eta\right) \bar{\triangleleft}\left(\langle\xi\rangle \triangleright u^{-1}\right) \otimes\left(\xi \bar{\triangleleft} u^{-1}\right)\right) .
$$


On the other hand,

$$
\begin{aligned}
u \dot{\triangleright} c(\chi(\eta) \otimes \chi(\xi)) & =u \dot{\triangleright} \chi\left(\left(\left(\langle\xi\rangle \triangleleft|\eta|^{-1}\right) \bar{\triangleright} \eta\right) \otimes \xi\right) \\
& =\chi\left(\left(\left(\left(\langle\xi\rangle \triangleleft|\eta|^{-1}\right) \bar{\triangleright} \eta\right) \otimes \xi\right) \bar{\triangleleft} u^{-1}\right),
\end{aligned}
$$

which gives the same as (10.28).

Now we show that $c$ preserves the $M$-action. For $s \in M$,

$$
\begin{aligned}
& s \dot{\triangleright}(\chi(\eta) \otimes \chi(\xi))=\chi\left(\left(s \triangleleft|\eta|^{-1}\right) \bar{\triangleright} \eta\right) \otimes \chi\left(\left(s \triangleleft|\xi|^{-1}\right) \bar{\triangleright} \xi\right), \\
& c(s \dot{\triangleright}(\chi(\eta) \otimes \chi(\xi)))=\chi\left(\left(\left\langle\left(s \triangleleft|\xi|^{-1}\right) \bar{\triangleright} \xi\right\rangle \triangleleft\left|\left(s \triangleleft|\eta|^{-1}\right) \bar{\triangleright} \eta\right|^{-1}\right) \bar{\triangleright}\left(\left(s \triangleleft|\eta|^{-1}\right) \bar{\triangleright} \eta\right)\right. \\
& \left.\otimes\left(\left(s \triangleleft|\xi|^{-1}\right) \bar{\triangleright} \xi\right)\right) \text {. }
\end{aligned}
$$

Using the "action" property for $\bar{\triangleright}$, we get

$$
\begin{gathered}
\left(\left\langle\left(s \triangleleft|\xi|^{-1}\right) \bar{\xi}\right\rangle \triangleleft\left|\left(s \triangleleft|\eta|^{-1}\right) \triangleright \eta\right|^{-1}\right) \overline{(}\left(\left(s \triangleleft|\eta|^{-1}\right) \triangleright \eta\right) \\
=\left(\left(p^{\prime} \cdot \bar{t}\right) \triangleright \eta\right) \triangleleft \tau\left(p^{\prime} \triangleleft(\bar{t} \triangleright|\eta|), \bar{t} \triangleleft|\eta|\right)^{-1},
\end{gathered}
$$

where $\bar{t}=s \triangleleft|\eta|^{-1}$ and

$$
p^{\prime}=\left\langle\left(s \triangleleft|\xi|^{-1}\right) \bar{\triangleright} \xi\right\rangle \triangleleft|\bar{t} \bar{\triangleright} \eta|^{-1} \tau(\langle\bar{t} \bar{\triangleright} \eta\rangle, \bar{t} \triangleleft|\eta|) \tau(\bar{t},\langle\eta\rangle)^{-1} .
$$

But, using the connections between the gradings and the actions, we know that $|\bar{t} \bar{\nabla} \eta|^{-1}=$ $(\bar{t} \triangleright|\eta|)^{-1} \boldsymbol{\tau}(\bar{t},\langle\eta\rangle) \tau(\langle\bar{t} \bar{\nabla}\rangle, \bar{t} \triangleleft|\eta|)^{-1}$, so

$$
\begin{aligned}
& p^{\prime}=\left\langle\left(s \triangleleft|\xi|^{-1}\right) \bar{\triangleright} \xi \triangleleft(\bar{t} \triangleright|\eta|)^{-1}\right. \\
& =\left\langle\left(s \triangleleft|\xi|^{-1}\right) \triangleright \xi\right\rangle \triangleleft\left(\left(s \triangleleft|\eta|^{-1}\right) \triangleright|\eta|\right)^{-1} \\
& =\left\langle\left(s \triangleleft|\xi|^{-1}\right) \bar{\triangleright} \xi\right\rangle \triangleleft\left(s \triangleright|\eta|^{-1}\right) \text {. }
\end{aligned}
$$

Substituting in the equation above gives

$$
\begin{aligned}
& \left(\left\langle\left(s \triangleleft|\xi|^{-1}\right) \triangleright \xi\right\rangle \triangleleft\left|\left(s \triangleleft|\eta|^{-1}\right) \bar{\triangleright} \eta\right|^{-1}\right) \bar{\Sigma}\left(\left(s \triangleleft|\eta|^{-1}\right) \triangleright \bar{\eta}\right) \\
& =\left(\left(\left(\left\langle\left(s \triangleleft|\xi|^{-1}\right) \bar{\triangleright} \xi\right\rangle \triangleleft\left(s \triangleright|\eta|^{-1}\right)\right) \cdot\left(s \triangleleft|\eta|^{-1}\right)\right) \triangleright \bar{\triangleright}\right) \bar{\triangleleft} \tau\left(\left\langle\left(s \triangleleft|\xi|^{-1}\right) \bar{\triangleright} \xi\right\rangle, s\right)^{-1} \\
& =\left(\left(\left(\left\langle\left(s \triangleleft|\xi|^{-1}\right) \bar{\triangleright} \xi\right\rangle \cdot s\right) \triangleleft|\eta|^{-1}\right) \bar{\triangleright} \eta\right) \bar{\triangleleft} \tau\left(\left\langle\left(s \triangleleft|\xi|^{-1}\right) \bar{\Sigma}\right\rangle, s\right)^{-1} \\
& =\left(\left(\left(\left(s \triangleleft|\xi|^{-1}\right) \cdot\langle\xi\rangle\right) \triangleleft|\eta|^{-1}\right) \bar{\triangleright} \eta\right) \triangleleft \tau\left(\left\langle\left(s \triangleleft|\xi|^{-1}\right) \bar{\triangleright} \xi\right\rangle, s\right)^{-1} \text {. }
\end{aligned}
$$

On the other hand, we know that

$$
\begin{aligned}
s \dot{\triangleright} c(\chi(\eta) \otimes \chi(\xi)) & =s \dot{\triangleright} \chi\left(\left(\left(\langle\xi\rangle \triangleleft|\eta|^{-1}\right) \bar{\triangleright} \eta\right) \otimes \xi\right) \\
& =s \dot{\triangleright} \chi(\bar{\eta} \otimes \xi)=\chi\left(\left(s \triangleleft|\bar{\eta} \otimes \xi|^{-1}\right) \bar{\triangleright}(\bar{\eta} \otimes \xi)\right),
\end{aligned}
$$


where $\bar{\eta}=\left(\langle\xi\rangle \triangleleft|\eta|^{-1}\right) \bar{\triangleright} \eta$. Next, we calculate

$$
\begin{aligned}
|\bar{\eta} \otimes \xi| & =\tau(\langle\bar{\eta}\rangle,\langle\xi\rangle)^{-1}|\bar{\eta}||\xi|, \\
s \triangleleft|\bar{\eta} \otimes \xi|^{-1} & =s \triangleleft|\xi|^{-1}|\bar{\eta}|^{-1} \tau(\langle\bar{\eta}\rangle,\langle\xi\rangle) .
\end{aligned}
$$

If we put $\bar{s}=s \triangleleft|\xi|^{-1}|\bar{\eta}|^{-1}$, then

$$
\begin{aligned}
(s \triangleleft \mid & \left.\left.\bar{\eta} \otimes \xi\right|^{-1}\right) \bar{\triangleright}(\bar{\eta} \otimes \xi) \\
& =(\bar{s} \triangleleft \tau(\langle\bar{\eta}\rangle,\langle\xi\rangle)) \bar{\triangleright}(\bar{\eta} \otimes \xi) \\
& =(\bar{s} \bar{\triangleright} \bar{\eta}) \bar{\triangleleft} \tau(\bar{s} \triangleleft|\bar{\eta}|,\langle\xi\rangle) \tau(\langle(\bar{s} \triangleleft|\bar{\eta}|) \bar{\triangleright} \xi\rangle, \bar{s} \triangleleft|\bar{\eta}||\xi|)^{-1} \otimes(\bar{s} \triangleleft|\bar{\eta}|) \triangleright \xi \\
& =(\bar{s} \bar{\triangleright} \bar{\eta}) \bar{\triangleleft} \tau\left(s \triangleleft|\xi|^{-1},\langle\xi\rangle\right) \tau\left(\left\langle\left(s \triangleleft|\xi|^{-1}\right) \bar{\triangleright} \xi\right\rangle, s\right)^{-1} \otimes\left(s \triangleleft|\xi|^{-1}\right) \bar{\triangleright} \xi .
\end{aligned}
$$

Using the "action" property again,

$$
\begin{aligned}
\bar{s} \bar{\triangleright} \bar{\eta} & =\left(s \triangleleft|\xi|^{-1}|\bar{\eta}|^{-1}\right) \bar{\triangleright}\left(\left(\langle\xi\rangle \triangleleft|\eta|^{-1}\right) \bar{\triangleright} \eta\right) \\
& =\left(\left(q^{\prime} \cdot\left(\langle\xi\rangle \triangleleft|\eta|^{-1}\right)\right) \bar{\triangleright} \eta\right) \bar{\triangleleft} \tau\left(q^{\prime} \triangleleft\left(\left(\langle\xi\rangle \triangleleft|\eta|^{-1}\right) \triangleright|\eta|\right),\langle\xi\rangle\right)^{-1} \\
& =\left(\left(q^{\prime} \cdot\left(\langle\xi\rangle \triangleleft|\eta|^{-1}\right)\right) \bar{\triangleright} \eta\right) \triangleleft \tau\left(q^{\prime} \triangleleft\left(\langle\xi\rangle \triangleright|\eta|^{-1}\right)^{-1},\langle\xi\rangle\right)^{-1},
\end{aligned}
$$

where

$$
\begin{aligned}
q^{\prime} & =\left(s \triangleleft|\xi|^{-1}|\bar{\eta}|^{-1}\right) \triangleleft \tau\left(\left\langle\left(\langle\xi\rangle \triangleleft|\eta|^{-1}\right) \triangleright \eta\right\rangle,\langle\xi\rangle\right) \tau\left(\langle\xi\rangle \triangleleft|\eta|^{-1},\langle\eta\rangle\right)^{-1} \\
& =\left(s \triangleleft|\xi|^{-1}\right) \triangleleft\left(\langle\xi\rangle \triangleright|\eta|^{-1}\right),
\end{aligned}
$$

as

$$
|\bar{\eta}|^{-1}=\left(\left(\langle\xi\rangle \triangleleft|\eta|^{-1}\right) \triangleright|\eta|\right)^{-1} \tau\left(\langle\xi\rangle \triangleleft|\eta|^{-1},\langle\eta\rangle\right) \tau\left(\left\langle\left(\langle\xi\rangle \triangleleft|\eta|^{-1}\right) \triangleright \eta\right\rangle,\langle\xi\rangle\right)^{-1} .
$$

Hence, substituting with the value of $q^{\prime}$, we get

$$
\begin{aligned}
\bar{s} \triangleright \bar{\eta} & =\left(\left(\left(\left(s \triangleleft|\xi|^{-1}\right) \triangleleft\left(\langle\xi\rangle \triangleright|\eta|^{-1}\right)\right) \cdot\left(\langle\xi\rangle \triangleleft|\eta|^{-1}\right)\right) \bar{\nabla}\right) \bar{\triangleleft} \tau\left(\left(s \triangleleft|\xi|^{-1}\right),\langle\xi\rangle\right)^{-1} \\
& =\left(\left(\left(\left(s \triangleleft|\xi|^{-1}\right) \cdot\langle\xi\rangle\right) \triangleleft|\eta|^{-1}\right) \triangleright \bar{\eta}\right) \triangleleft \tau\left(s \triangleleft|\xi|^{-1},\langle\xi\rangle\right)^{-1},
\end{aligned}
$$

giving the required result

$$
\begin{aligned}
& (\bar{s} \triangleright \bar{\eta}) \triangleleft \tau\left(s \triangleleft|\xi|^{-1},\langle\xi\rangle\right) \tau\left(\left\langle\left(s \triangleleft|\xi|^{-1}\right) \triangleright \xi\right\rangle, s\right)^{-1} \\
& =\left(\left(\left(\left(s \triangleleft|\xi|^{-1}\right) \cdot\langle\xi\rangle\right) \triangleleft|\eta|^{-1}\right) \triangleright \eta\right) \triangleleft \tau\left(\left\langle\left(s \triangleleft|\xi|^{-1}\right) \bar{\triangleright} \xi\right\rangle, s\right)^{-1} .
\end{aligned}
$$

\section{REFERENCES}

[1] B. Bakalov and A. Kirillov Jr., Lectures on Tensor Categories and Modular Functors, University Lecture Series, vol. 21, American Mathematical Society, Rhode Island, 2001.

[2] E. J. Beggs, Making non-trivially associated tensor categories from left coset representatives, J. Pure Appl. Algebra 177 (2003), no. 1, 5-41.

[3] E. J. Beggs, J. D. Gould, and S. Majid, Finite group factorizations and braiding, J. Algebra 181 (1996), no. 1, 112-151. 
[4] E. J. Beggs and S. Majid, Quasitriangular and differential structures on bicrossproduct Hopf algebras, J. Algebra 219 (1999), no. 2, 682-727.

[5] L. C. Grove, Groups and Characters, Pure and Applied Mathematics, John Wiley \& Sons, New York, 1997.

[6] S. Majid, Foundations of Quantum Group Theory, Cambridge University Press, Cambridge, 1995.

[7] A. D. Thomas and G. V. Wood, Group Tables, Shiva Mathematics Series, vol. 2, Shiva Publishing, Nantwich, 1980.

[8] V. Turaev and H. Wenzl, Semisimple and modular categories from link invariants, Math. Ann. 309 (1997), no. 3, 411-461.

M. M. Al-Shomrani: Department of Mathematics, University of Wales, Swansea, Singleton Park, SA2 8PP, UK

E-mail address: ma1 shomrani@hotmai 1 .com

E. J. Beggs: Department of Mathematics, University of Wales, Swansea, Singleton Park, SA2 8PP, UK

E-mail address: e.j .beggs@swansea.ac.uk 


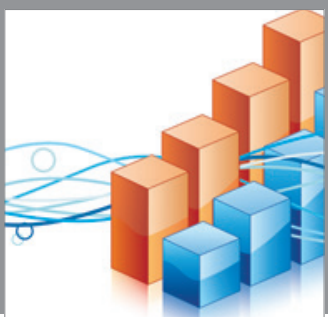

Advances in

Operations Research

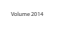

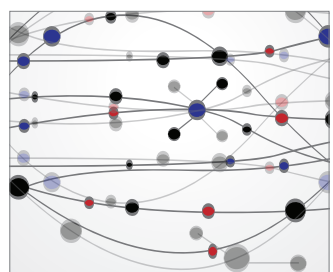

\section{The Scientific} World Journal
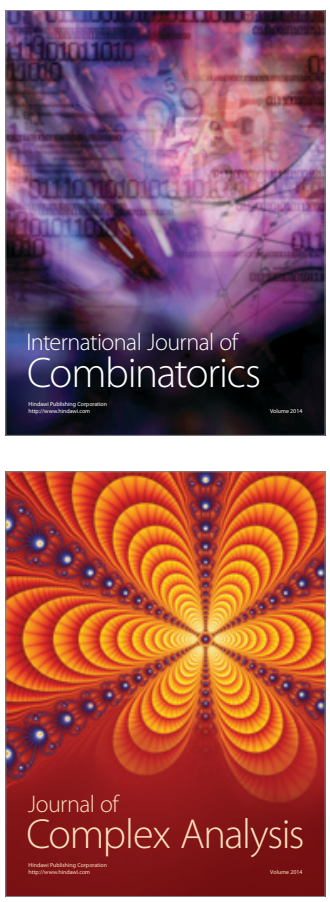

International Journal of

Mathematics and

Mathematical

Sciences
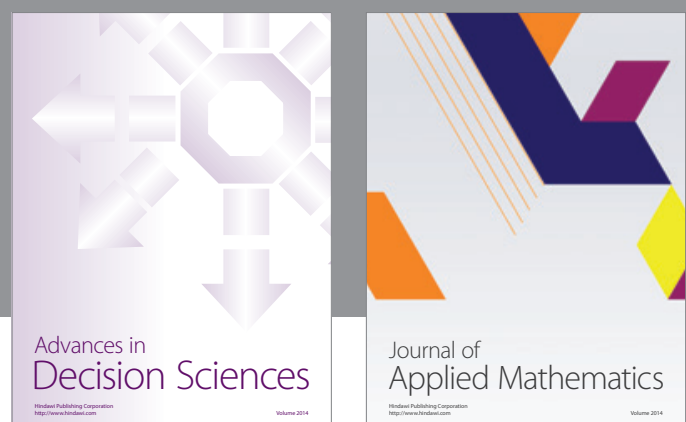

Journal of

Applied Mathematics
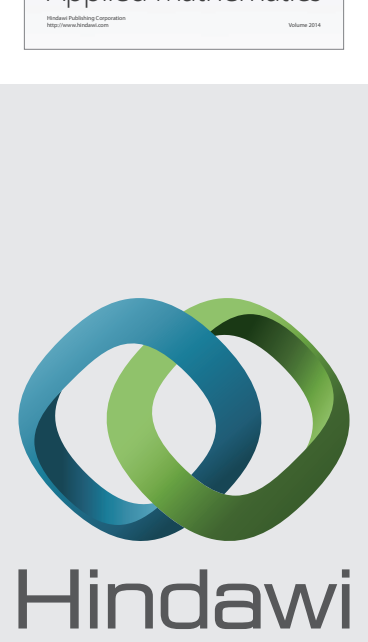

Submit your manuscripts at http://www.hindawi.com
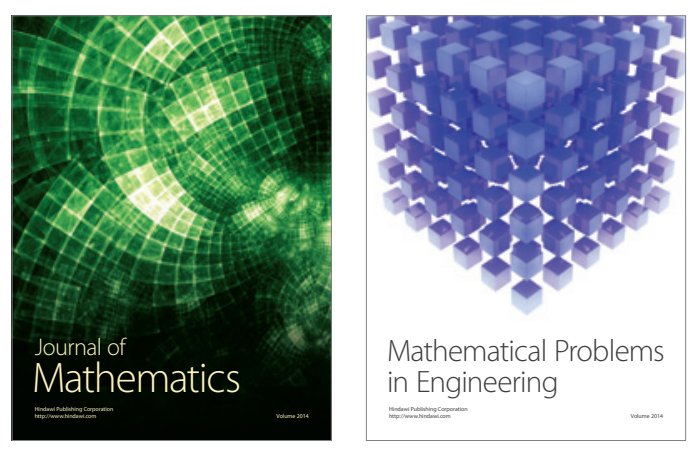

Mathematical Problems in Engineering
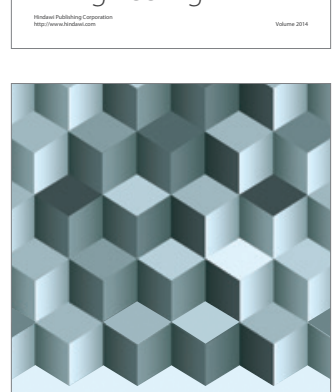

Journal of

Function Spaces
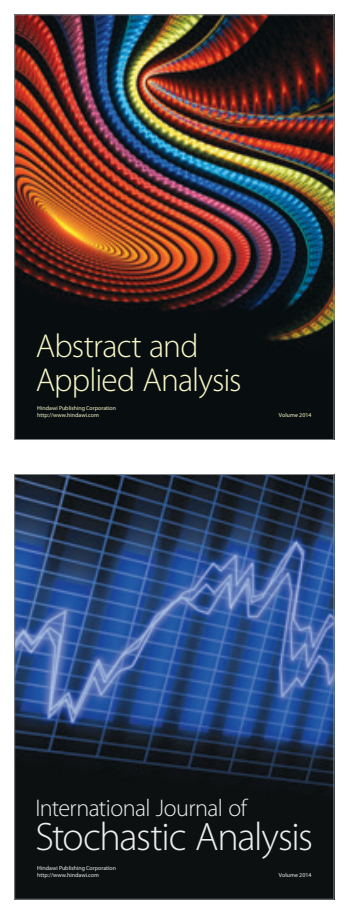

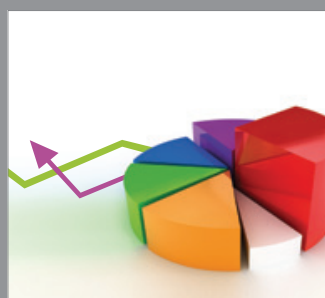

ournal of

Probability and Statistics

Promensencen
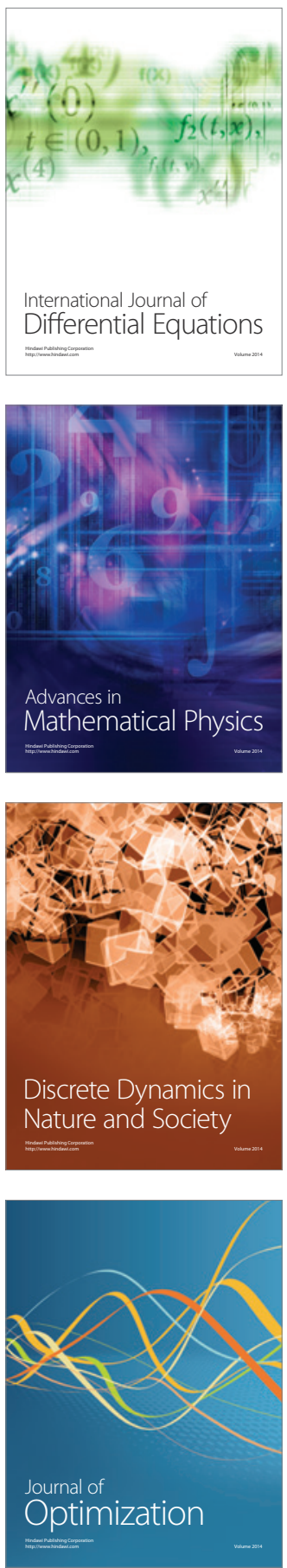